\title{
Current Clinical Strategies of Pancreatic Cancer Treatment and Open Molecular Questions
}

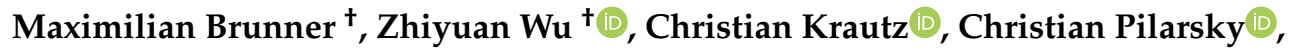 \\ Robert Grützmann and Georg F. Weber* \\ Department of General and Visceral Surgery, Friedrich Alexander University, Krankenhausstraße 12, \\ 91054 Erlangen, Germany; Maximilian.Brunner@uk-erlangen.de (M.B.); zhiyuan.wu@fau.de (Z.W.); \\ Christian.Krautz@uk-erlangen.de (C.K.); Christian.Pilarsky@uk-erlangen.de (C.P.); \\ Robert.Gruetzmann@uk-erlangen.de (R.G.) \\ * Correspondence: Georg.weber@uk-erlangen.de; Tel.: +49-9131-8533296 \\ + These authors contributed equally to this manuscript.
}

Received: 9 August 2019; Accepted: 12 September 2019; Published: 13 September 2019

\begin{abstract}
Pancreatic cancer is one of the most lethal malignancies and is associated with a poor prognosis. Surgery is considered the only potential curative treatment for pancreatic cancer, followed by adjuvant chemotherapy, but surgery is reserved for the minority of patients with non-metastatic resectable tumors. In the future, neoadjuvant treatment strategies based on molecular testing of tumor biopsies may increase the amount of patients becoming eligible for surgery. In the context of non-metastatic disease, patients with resectable or borderline resectable pancreatic carcinoma might benefit from neoadjuvant chemo- or chemoradiotherapy followed by surgeryPatients with locally advanced or (oligo-/poly-)metastatic tumors presenting significant response to (neoadjuvant) chemotherapy should undergo surgery if R0 resection seems to be achievable. New immunotherapeutic strategies to induce potent immune response to the tumors and investigation in molecular mechanisms driving tumorigenesis of pancreatic cancer may provide novel therapeutic opportunities in patients with pancreatic carcinoma and help patient selection for optimal treatment.
\end{abstract}

Keywords: pancreatic cancer; treatment options; surgery; chemotherapy; chemoradiation; therapeutic targets; molecular mechanism; immunotherapy

\section{Introduction}

Pancreatic cancer is one of the most lethal malignancies, accounting for the 7th leading cause of cancer-related mortality worldwide [1]. It is estimated that about 458,000 people will be diagnosed with pancreatic cancer worldwide in 2018, and more than 432,000 will die of this disease. 5-year survival in patients with pancreatic cancer is as low as $9 \%$ in the USA [2]. Although progress has been made in multimodality treatment with surgery and adjuvant therapy, the mortality rate of pancreatic cancer is still increasing throughout the years. The disappointing prognosis of this disease is largely attributable to its late diagnosis, as most patients with pancreatic cancer remain asymptomatic until the disease develops to an advanced stage [3]. Only non-specific symptoms may exist at early stage, but there are currently no screening programs available [4]. Besides, tumor biology of pancreatic cancer may contribute to its early metastasis. A preclinical study using a mouse model of pancreatic cancer indicates that early metastasis might possibly be detected even when there is no primary tumor found in the pancreas and is associated with epithelial-to-mesenchymal transition and focal inflammation [5]. Therefore, like many other types of cancers, pancreatic cancer is suggested to be a systematic disease, and multidisciplinary management of this disease is of great importance. Treatment of pancreatic 
cancers includes surgery, chemotherapy, radiation therapy, and palliative care, which are selected on the basis of disease stage (Figure 1). Here we review the current clinical strategies in the treatment of pancreatic ductal adenocarcinoma (PDAC), the most common type of pancreatic cancers, in different scenarios (resectable, borderline resectable, locally advanced, and metastatic PDAC), and potential novel approaches under development based on the expanding molecular biology knowledge.

(a)

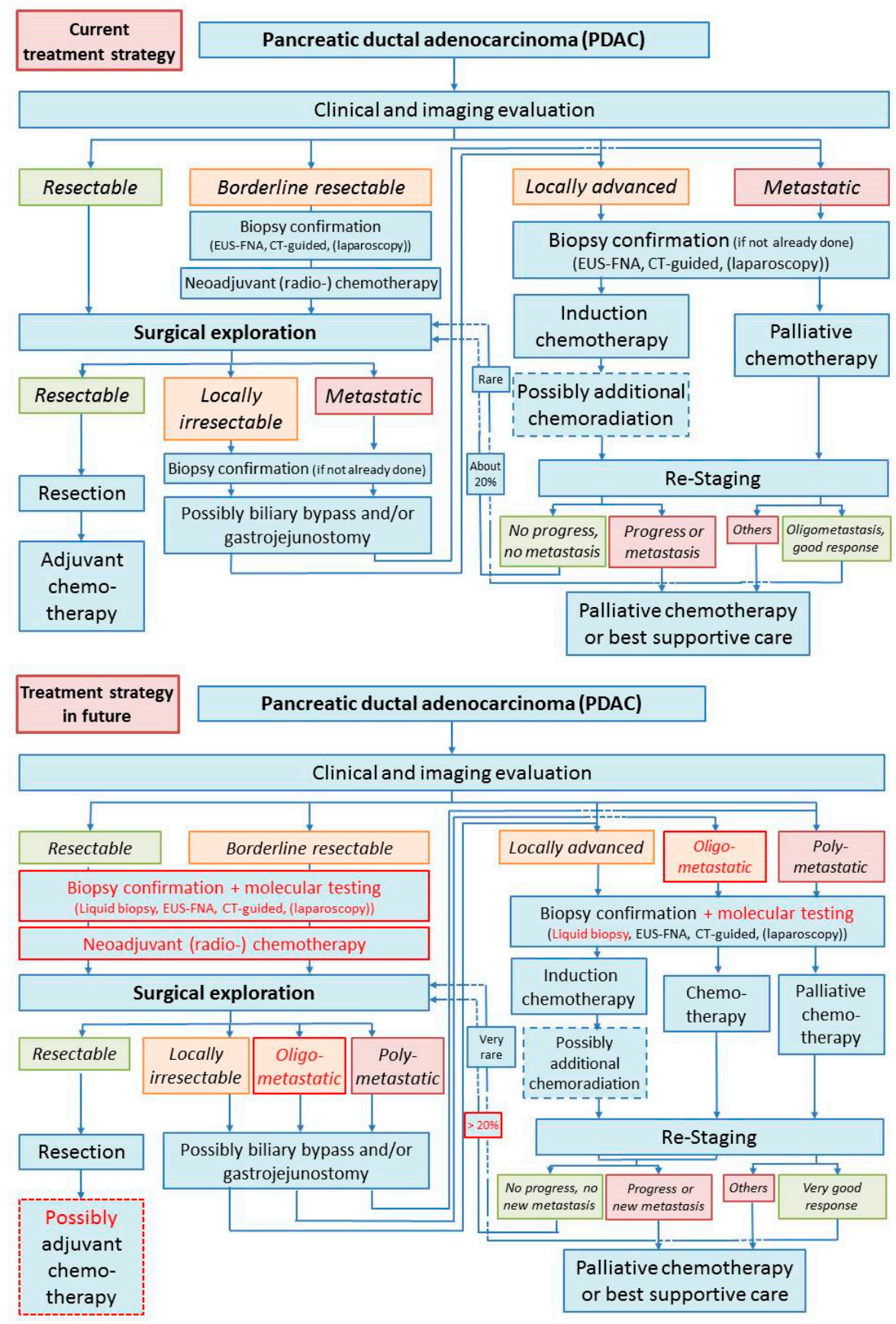

Figure 1. (a) Current treatment strategies for pancreatic carcinomas; (b) assumed future treatment strategy for pancreatic carcinoma: The most distinctive changes (highlighted in red) are probably (1) an extension of biopsy options and an introduction of routinely molecular tests including chemotherapy sensitivity, (2) an introduction of neoadjuvant therapy even in resectable stage, (3) an improvement of chemotherapy regimens with increased secondary resectability, and (4) the "introduction" of oligometastasis as the fifth subgroup (besides resectable, borderline, locally advanced, and metastatic stages) with enhanced therapy options including surgery in this new group. Dotted frames indicate possible therapy options. 


\section{Current Management of Pancreatic Cancer}

\subsection{Management of Resectable Pancreatic Cancers}

In the management of pancreatic carcinomas, surgical resection is fundamental as surgery is currently the only potential treatment to cure pancreatic cancer that could result in significant improvement in survival, but over $80 \%$ of patients with pancreatic cancer are diagnosed when the lesion is no longer primarily resectable $[3,6,7]$. Therefore, upfront surgery is currently standard in patients with resectable pancreatic carcinomas. The most important goal of surgical resection of pancreatic cancer is to achieve a negative resection margin (R0), as positive resection margin (R1 or R2) is associated with recurrence and dismal prognosis [8,9]. Achieving an R0 margin mandates meticulous perivascular dissection, recognition of the need for vascular resection and reconstruction, as well as potential need for extra-pancreatic organ resection. Additionally, pancreatic cancer surgery is strongly recommended to be performed in specialized high-volume centers, as the hospital volume is reported by many groups associated with resection margin, in-hospital mortality, and long-term survival [10-12].

Adjuvant chemotherapy is generally considered, as most patients will develop recurrence after surgical resection. There have been some robust randomized clinical trials evaluating the role of different postoperative chemotherapy regimens, especially for 5-fluorouracil, gemcitabine and combination regimen (e.g., FOLFIRINOX) (Table 1) [13-30]. Currently FOLFIRINOX provides the standard adjuvant therapy in patients with high performance status. The effectiveness of postoperative chemoradiotherapy (CRT) remains controversial. The supportive evidence of the use of adjuvant CRT mainly comes from retrospective cohort studies [31-34]. However, a pooled analysis of nine randomized controlled trials showed that adjuvant CRT results in worse survival than chemotherapy alone [35]. High-quality clinical evidence from randomized controlled trials is limited at present.

Table 1. Summary of randomized controlled trials concerning adjuvant chemotherapy in patients with pancreatic carcinomas.

\begin{tabular}{|c|c|c|c|c|c|}
\hline Trial & Year & Country/Region & $\mathbf{N}$ & Regimens & Survival Outcomes \\
\hline Bakkevold [13] & 1993 & Norway & 47 & $\begin{array}{l}\text { AMF (5-FU, doxorubicin, } \\
\text { mitomycin C) }(n=23) \text { vs. } \\
\text { observation }(n=24)\end{array}$ & $\begin{array}{c}\text { mOS } 23 \text { mo vs. } 11 \text { mo }(p=0.02) \\
\text { 2-year survival } 43 \% \text { vs. } 32 \% ; \\
\text { 5-year survival } 4 \% \text { vs. } 8 \%\end{array}$ \\
\hline Takada [14] & 2002 & Japan & 158 & $\begin{array}{l}\text { MF (5-FU and mitomycin C) }(n \\
=81) \text { vs. observation }(n=77)\end{array}$ & $\begin{array}{c}5 \text {-year OS } 17.8 \% \text { vs. } 26.6 \% \text { in } \\
\text { patients with curative resection } \\
(p=0.4544) ; 5 \text {-year DFS } 8.6 \% \text { vs. } \\
7.8 \%(p=0.8372)\end{array}$ \\
\hline ESPAC-1 [15] & 2004 & Europe & 289 & $\begin{array}{l}\text { 5-FU/folinic acid (with and } \\
\text { without chemoradiotherapy) }(n \\
=147) \text { vs. no chemotherapy } \\
\text { (observation and } \\
\text { chemoradiotherapy) }(n=142)\end{array}$ & $\begin{array}{c}\text { mOS } 20.1 \text { mo vs. } 15.5 \text { mo ( } p= \\
0.009) ; 2 \text {-year estimated OS } 40 \% \\
\text { vs. } 30 \% \text {; } 5 \text {-year estimated OS } \\
21 \% \text { vs. } 8 \% \text {; mDFS } 15.3 \text { mo vs. } \\
9.4 \text { mo ( } p=0.02) ; 1 \text {-year DFS } \\
58 \% \text { vs. } 43 \%\end{array}$ \\
\hline JSAP [16] & 2006 & Japan & 89 & $\begin{array}{l}\text { 5-FU/cisplatin }(n=45) \text { vs. } \\
\text { observation }(n=44)\end{array}$ & $\begin{array}{c}\text { mOS } 12.5 \text { mo vs. } 15.8 \text { mo; } 5 \text {-year } \\
\text { OS } 26.4 \% \text { vs. } 14.9 \%\end{array}$ \\
\hline $\begin{array}{c}\text { CONKO-001 } \\
{[17,18]}\end{array}$ & $\begin{array}{l}2007 \\
\text { and } \\
2013\end{array}$ & $\begin{array}{l}\text { Germany and } \\
\text { Austria }\end{array}$ & 368 & $\begin{array}{l}\text { Gemcitabine } 6 \text { cycles }(n=179) \\
\text { vs. observation }(n=175)\end{array}$ & $\begin{array}{c}\text { mOS } 22.8 \text { mo vs. } 20.2 \text { mo ( } p= \\
\text { 0.01); } 5 \text {-year OS } 20.7 \% \text { vs. } 10.4 \% \text {; } \\
\text { 10-year OS } 12.2 \% \text { vs. } 7.7 \% \text {; } \\
\text { mDFS } 13.4 \text { mo vs. } 6.9 \text { mo ( } p< \\
\text { 0.001); } 3 \text {-year DFS } 23.5 \% \text { vs. } \\
7.5 \% \text {; } 5 \text {-year DFS } 16.5 \% \text { vs. } 5.5 \%\end{array}$ \\
\hline
\end{tabular}


Table 1. Cont.

\begin{tabular}{|c|c|c|c|c|c|}
\hline Trial & Year & Country/Region & $\mathbf{N}$ & Regimens & Survival Outcomes \\
\hline Yoshitomi [19] & 2008 & Japan & 100 & $\begin{array}{l}\text { Gemcitabine and UFT } \\
\text { (uracil/tegafur) }(n=50) \text { vs. } \\
\text { gemcitabine }(n=49)\end{array}$ & $\begin{array}{c}\text { mOS } 21.2 \text { mo vs. } 29.8 \mathrm{mo}(p= \\
0.28) ; 1-\text { year OS } 80.0 \% \text { vs. } 85.7 \% \text {; } \\
\text { 3-year OS } 30.4 \% \text { vs. } 46.9 \% ; \\
\text { mDFS } 12.3 \text { mo vs. } 12.0 \text { mo ( } p= \\
\text { 0.67); } 1 \text {-year DFS } 50.0 \% \text { vs. } \\
49.0 \% \text {; } 3 \text {-year DFS } 17.7 \% \\
\text { vs. } 21.6 \%\end{array}$ \\
\hline $\begin{array}{l}\text { ESPAC-1 plus } \\
\text { [20] }\end{array}$ & 2009 & Europe & 192 & $\begin{array}{l}\text { 5-FU/folinic acid }(n=97) \text { vs. } \\
\quad \text { observation }(n=95)\end{array}$ & $\begin{array}{c}\text { mOS } 24.0 \text { mo vs. } 12.8 \text { mo; } 2 \text {-year } \\
\text { OS } 49 \% \text { vs. } 28 \% ; 5 \text {-year OS } 24 \% \\
\text { vs. } 14 \%\end{array}$ \\
\hline $\begin{array}{l}\text { ESPAC-3 v1 } \\
{[20]}\end{array}$ & 2009 & Europe & 122 & $\begin{array}{l}\text { 5-FU/folinic acid }(n=61) \mathrm{vs.} \\
\quad \text { observation }(n=61)\end{array}$ & $\begin{array}{c}\text { mOS } 25.9 \text { mo vs. } 20.3 \text { mo; } 2 \text {-year } \\
\text { OS } 54 \% \text { vs. } 48 \% ; 5 \text {-year OS } 20 \% \\
\text { vs. } 20 \%\end{array}$ \\
\hline JSAP-02 [21] & 2009 & Japan & 119 & $\begin{array}{c}\text { Gemcitabine } 3 \text { cycles }(n=58) \text { vs. } \\
\text { observation }(n=60)\end{array}$ & $\begin{array}{l}\text { mOS } 22.3 \text { mo vs. } 18.4 \text { mo }(p= \\
0.19) ; \text { mDFS } 11.4 \text { mo vs. } 5.0 \text { mo } \\
(p=0.01)\end{array}$ \\
\hline $\begin{array}{c}\text { ESPAC-3 v2 } \\
\text { [22] }\end{array}$ & 2010 & International & 1088 & $\begin{array}{l}\text { 5-FU/folinic acid }(n=551) \text { vs. } \\
\text { gemcitabine }(n=537) \text { for } 6 \text { mo }\end{array}$ & $\begin{array}{c}\text { mOS } 23.0 \text { mo vs. } 23.6 \text { mo ( } p= \\
0.39) ; \text { estimated } 2 \text {-year OS } 48.1 \% \\
\text { vs. } 49.1 \% \text {; mPFS } 14.1 \text { mo vs. } \\
14.3 \text { mo }(p=0.53) \text {; estimated } \\
\text { 2-year PFS } 30.7 \% \text { vs. } 29.6 \%\end{array}$ \\
\hline $\begin{array}{l}\text { RTOG 97-04 } \\
{[23,24]}\end{array}$ & 2011 & $\begin{array}{l}\text { USA and } \\
\text { Canada }\end{array}$ & 451 & $\begin{array}{l}\text { 5-FU }(n=230) \text { vs. gemcitabine } \\
(n=221), \text { both with (before and } \\
\text { after) CRT (5-FU and } 50.4 \mathrm{~Gy})\end{array}$ & $\begin{array}{l}\text { For pancreatic head tumors, } \\
\text { mOS } 17.1 \text { mo vs. } 20.5 \text { mo ( } p= \\
0.12) ; 5 \text {-year OS } 18 \% \text { vs. } 22 \%\end{array}$ \\
\hline PACT-7 [25] & 2012 & $\begin{array}{l}\text { Italy and } \\
\text { Switzerland }\end{array}$ & 102 & $\begin{array}{l}\text { Gemcitabine }(n=51) \text { vs. PEFG } \\
\text { (cisplatin, epirubicin, 5-FU, } \\
\text { gemcitabine) }(n=49), \text { both } \\
\text { followed by chemoradiation } \\
\text { (5-FU and 54-60 Gy) }\end{array}$ & $\begin{array}{c}\text { mOS } 24.8 \text { mo vs. } 28.9 \text { mo; mDFS } \\
11.7 \text { mo vs. } 15.2 \text { mo; } 1 \text {-year DFS } \\
49.0 \% \text { vs. } 69.4 \%\end{array}$ \\
\hline Shimoda [26] & 2015 & Japan & 57 & $\begin{array}{l}\text { S-1 }(n=29) \text { vs. gemcitabine } \\
\qquad(n=28)\end{array}$ & $\begin{array}{l}\text { mOS } 21.5 \text { mo vs. } 18.0 \text { mo ( } p= \\
0.293) ; 2 \text {-year OS } 46 \% \text { vs. } 38 \% \\
\text { mDFS } 14.6 \text { mo vs. } 10.5 \text { mo ( } p= \\
0.188 \text { ); } 2 \text {-year DFS } 41 \% \text { vs. } 18 \%\end{array}$ \\
\hline JASPAC 01 [27] & 2016 & Japan & 385 & $\begin{array}{l}\text { S-1 }(n=187) \text { vs. gemcitabine } \\
(n=190)\end{array}$ & $\begin{array}{c}\text { mOS } 46.5 \text { mo vs. } 25.5 \text { mo }(p< \\
0.0001) ; 5 \text {-year OS } 44.1 \% \text { vs. } \\
24.4 \% \text {; mRFS } 22.9 \text { mo vs. } 11.3 \\
\text { mo }(p<0.0001) ; 5 \text {-year RFS } \\
33.3 \% \text { vs. } 16.8 \% ; \text { recurrence } 66 \% \\
\text { vs. } 78 \%\end{array}$ \\
\hline ESPAC-4 [28] & 2017 & Europe & 732 & $\begin{array}{l}\text { Gemcitabine and capecitabine }(n \\
=364) \text { vs. gemcitabine }(n=366)\end{array}$ & $\begin{array}{c}\text { mOS } 28.0 \text { mo vs. } 25.5 \text { mo ( } p= \\
0.032) \text {; estimated 1-year OS } \\
84.1 \% \text { vs. } 80.5 \% \text {; estimated } \\
\text { 2-year OS } 53.8 \% \text { vs. } 52.1 \% \text {; in R1 } \\
\text { patients, mOS } 23.7 \text { mo vs. } 23.0 \\
\text { mo; in R0 patients, mOS } 39.5 \text { mo } \\
\text { vs. } 27.9 \text { mo ( } p=0.0001)\end{array}$ \\
\hline $\begin{array}{c}\text { CONKO-005 } \\
{[29]}\end{array}$ & 2017 & Germany & 436 & $\begin{array}{l}\text { Gemcitabine and erlotinib }(n= \\
219) \text { vs. gemcitabine }(n=217)\end{array}$ & $\begin{array}{c}\text { mOS } 24.5 \text { mo vs. } 26.5 \text { mo ( } p= \\
0.61) \text {; estimated } 2 \text {-year OS } 53 \% \\
\text { vs. } 54 \% \text {; estimated } 5 \text {-year OS } \\
23 \% \text { vs. } 20 \% \text {; mDFS } 11.4 \text { mo vs. } \\
11.4 \text { mo }(p=0.26) ; \text { estimated } \\
\text { 2-year DFS } 25 \% \text { vs. } 25 \% ; \\
\text { estimated } 5 \text {-year DFS } 12 \% \\
\text { vs. } 11 \%\end{array}$ \\
\hline PACT-15 [30] & 2018 & Italy & 93 & $\begin{array}{c}\text { Gemcitabine } 6 \text { cycles }(n=26) \text { vs. } \\
\text { PEXG (gemcitabine, cisplatin, } \\
\text { epirubicin, capecitabine) } 6 \\
\text { cycles }(n=30)\end{array}$ & $\begin{array}{c}\text { mOS } 20.4 \text { mo vs. } 26.4 \text { mo; } 3 \text {-year } \\
\text { OS } 35 \% \text { vs. } 43 \% \text {; } 5 \text {-year OS } 13 \% \\
\text { vs. } 24 \% \text {; mDFS } 4.7 \text { mo vs. } 12.4 \\
\text { mo; } 1 \text {-year DFS } 23 \% \text { vs. } 50 \%\end{array}$ \\
\hline
\end{tabular}


Due to lacking evidence, preoperative neoadjuvant treatment could not be recommended for resectable pancreatic cancers but is investigated currently in randomized controlled studies for primary resectable pancreatic carcinoma (Table 2) [30,36-40].

Table 2. Summary of randomized controlled trials concerning neoadjuvant chemo(radio-)therapy in patients with pancreatic carcinomas.

\begin{tabular}{|c|c|c|c|c|c|}
\hline Trial & Year & Country/Region & $\mathbf{N}$ & Regimens & Outcomes \\
\hline \multicolumn{6}{|c|}{ Neoadjuvant chemotherapy } \\
\hline Palmer [36] & 2007 & UK & $\begin{array}{l}\text { 50, resectable } \\
\text { PC }\end{array}$ & $\begin{array}{l}\text { Gemcitabine }(n=24) \text { vs. } \\
\text { gemcitabine and } \\
\text { cisplatin }(n=26)\end{array}$ & $\begin{array}{c}\text { Resection rate } 38 \% \text { vs. } \\
70 \% \text {; R0 resection } 75 \% \text { vs. } \\
75 \% \text {; mOS } 9.9 \text { mo vs. } 15.6 \\
\text { mo; 1-year OS } 41.7 \% \text { vs. } \\
61.5 \%\end{array}$ \\
\hline Sahora [37] & 2014 & Austria & $\begin{array}{c}30,11 x \\
\text { borderline } \\
\text { resectable and } \\
19 x \text { locally } \\
\text { advanced }\end{array}$ & $\begin{array}{l}\text { Gemcitabine } 4 \text { cycles and } \\
\text { bevacizumab } 3 \text { doses }(n \\
=11) \text { vs. gemcitabine } 4 \\
\text { cycles and bevacizumab } \\
6 \text { doses }(n=19)\end{array}$ & $\begin{array}{c}\text { resection rate } 36.4 \% \text { vs. } \\
36.8 \%(p=0.97)\end{array}$ \\
\hline PACT-15 [30] & 2018 & Italy & 93 & $\begin{array}{l}\text { PEXG (gemcitabine, } \\
\text { cisplatin, epirubicin, } \\
\text { capecitabine) } 3 \text { cycles } \\
\text { before and after surgery } \\
(n=29) \text { vs. PEXG } 6 \\
\text { cycles after surgery }\end{array}$ & $\begin{array}{c}\text { mOS } 38.2 \text { mo vs. } 26.4 \text { mo; } \\
\text { 3-year OS } 55 \% \text { vs. } 43 \% ; \\
5 \text {-year OS } 49 \% \text { vs. } 24 \% \text {; } \\
\text { mDFS } 16.9 \text { mo vs. } 12.4 \text { mo; } \\
\text { 1-year DFS } 66 \% \text { vs. } 50 \%\end{array}$ \\
\hline \multicolumn{6}{|c|}{ Neoadjuvant CRT } \\
\hline E1200 [38] & 2010 & USA & 23 & $\begin{array}{l}\text { CRT (gemcitabine and } \\
50.4 \mathrm{~Gy})(n=10) \text { vs. } \\
\text { chemotherapy } \\
\text { (gemcitabine, cisplatin, } \\
5 \text {-FU) followed by CRT } \\
\text { (5-FU, } 50.4 \mathrm{~Gy})(n=11), \\
\text { both followed by surgery } \\
\text { and gemcitabine } \\
\text { adjuvant chemotherapy }\end{array}$ & $\begin{array}{c}\text { mOS } 19.4 \text { mo vs. } 13.4 \text { mo; } \\
\text { 1-year acturial OS } 69 \% \text { vs. } \\
61 \% \text {; } 2 \text {-year acturial OS } \\
32 \% \text { vs. } 13 \% \text {; mPFS } 14.2 \\
\text { mo vs. not given; } 1 \text {-year } \\
\text { acturial PFS } 59 \% \text { vs. } 15 \% \text {; } \\
\text { resectability } 30 \% \text { vs. } 18.2 \%\end{array}$ \\
\hline Golcher [39] & 2015 & $\begin{array}{l}\text { Germany and } \\
\text { Switzerland }\end{array}$ & 73 & $\begin{array}{l}\text { Upfront surgery }(n=33 \text { ) } \\
\text { vs. neoadjuvant CRT } \\
\text { (gemcitabine, cisplatin, } \\
\text { and } 55.8 \text { Gy) followed by } \\
\text { surgery }(n=33), \text { both } \\
\text { followed by gemcitabine } \\
\text { chemotherapy }\end{array}$ & $\begin{array}{c}\text { mOS } 14.4 \text { mo vs. } 17.4 \text { mo } \\
(p=0.96) ; \mathrm{mPFS} 8.7 \text { mo vs. } \\
8.4 \text { mo }(p=0.95) ; \mathrm{R} 0 \text { rate } \\
48 \% \text { vs. } 52 \%(p=0.81) ; \\
\text { pN0 rate } 30 \% \text { vs. } 39 \% \\
(p=0.44)\end{array}$ \\
\hline Casadei [40] & 2015 & Italy & 38 & $\begin{array}{l}\text { Upfront surgery }(n=20) \\
\text { vs. neoadjuvant CRT } \\
\text { (gemcitabine) followed } \\
\text { by surgery }(n=18), \text { both } \\
\text { followed by gemcitabine } \\
\text { chemotherapy }\end{array}$ & $\begin{array}{c}\text { mOS } 19.5 \text { mo vs. } 22.4 \text { mo } \\
(p=0.973) ; \text { resectability } \\
75 \% \text { vs. } 61.1 \%(p=0.489) \\
\text { R0 rate } 25.0 \% \text { vs. } 38.9 \% \\
(p=0.489)\end{array}$ \\
\hline
\end{tabular}

There is currently no reliable clinical evidence or expert consensus to recommend any surveillance strategies after potential curative resection of pancreatic cancer. A retrospective study showed that postoperative surveillance with clinical evaluation and serum carbohydrate antigen (CA) 19-9, together with routine $\mathrm{CT}$ and chest $\mathrm{X}$-ray, for every 6 months, is effective and associated with a better survival outcome; however, increased frequency and intensity of surveillance does not bring any benefit [41,42].

For patients experiencing recurrence following resection, the choice of second-line therapies will depend on the site of recurrent disease. Patients with local recurrence will potentially be subjected to surgical resection (if recurrence is in the pancreas or locoregional lymph nodes only) or chemoradiation. Unfortunately, however, most patients with recurrent disease develop metastasis, where systemic chemotherapy may be considered. In rare cases of isolated and limited liver metastasis, resection can 
improve survival. In addition, palliative and best supportive care is another option, especially for patients with poor performance status.

\subsection{Management of Borderline Resectable Pancreatic Cancers}

In patients with suspected borderline resectable disease, biopsy confirmation of adenocarcinoma with endoscopic ultrasound-guided fine needle aspiration (EUS-FNA), CT-guided biopsy, or staging laparoscopy is required.

After ruling out metastatic disease, neoadjuvant chemotherapy or chemoradiotherapy is applied, preferentially within a clinical trial. In the last years, preoperative neoadjuvant therapy was investigated in order to obtain better local control and eliminate potential micrometastasis of the disease. A recent systematic review of 35 comparative studies demonstrated that neoadjuvant therapy could improve resectability of the disease through down-staging of the tumor, especially in borderline pancreatic cancers [43]. Several large retrospective cohort studies based on a national cancer database also demonstrated that preoperative chemotherapy was associated with improved survival outcome in resected pancreatic cancers of different pathological stages [44,45]. In addition, another systematic review indicated that neoadjuvant therapy is safe and does not affect postoperative complication rates [46]. However, there are currently very few results from randomized trials available with limited quality of evidence (Table 2) [30,36-40]. No clear conclusion could be drawn whether there is advantage of neoadjuvant CRT compared to chemotherapy alone. Many of the larger phase 3 randomized controlled trials are prematurely terminated because of failure of recruitment, as the patients may fear losing the opportunity for surgical resection. Nonetheless, there are still many randomized trials ongoing to explore the potential effects of CRT. For instance, a large phase 3 trial, CONKO-007, was proposed to demonstrate, with 830 participants expected, the effect of CRT as compared to chemotherapy alone in the neoadjuvant setting, which is awaited to disclose meaningful evidence to this field.

After neoadjuvant treatment, surgical exploration is considered with preferable tumor response detected by restaging imaging assessment. Then, resection or palliative operative procedures could be performed depending on the intraoperative findings.

Similar to primary resectable tumors, adjuvant therapies are generally considered as a result of the high risk of recurrence even after radical surgical resection and the association of adjuvant chemotherapy with improved survival in patients with resected pancreatic adenocarcinoma (Table 1) [13-30,35,44].

\subsection{Management of Locally Advanced and Metastatic Pancreatic Cancer}

Unresectable pancreatic cancers, both locally advanced and metastatic, might be detected with imaging for staging and resectability assessment or discovered during surgical exploration for patients initially considered as potentially resectable. After biopsy confirmation, the patient's performance status is evaluated, where patients with good performance status might probably be able to tolerate more aggressive treatment. There have been extensive investigations on various chemotherapeutic regimens, which are basically classified into fluoropyrimidine- and gemcitabine-based regimens (Table 3) [47-74]. If the patient's performance status is good (usually determined as Eastern Co-operative of Oncology Group (ECOG) scores 0-1), various combination chemotherapy, including fluoropyrimidine- and gemcitabine-based regimens, are recommended, as a recent Cochrane analysis shows that a combination of several chemotherapeutic agents in advanced pancreatic carcinoma is superior to gemcitabine alone, albeit with a higher side effect profile [75]. Especially the FOLFIRINOX regimen is strongly recommended for patients with locally advanced or metastatic disease. Other combination regimens, especially gemcitabine-based, are usually used for patients with metastatic patients unlikely to tolerate FOLFIRINOX. However, based on the current evidence, it is not possible to determine the optimal gemcitabine combination. The combination of gemcitabine and erlotinib has been associated with significant improvement in overall and progression free survival as compared with gemcitabine alone, although this improvement was small. One characteristic adverse effect of the use of erlotinib is 
skin rash, and patients who developed skin rash after use of erlotinib might probably have better survival outcomes [56,73]. For patients not fit enough to tolerate combination regimens, gemcitabine monotherapy is considered.

Table 3. Summary of randomized controlled trials concerning first-line chemotherapy in patients with locally advanced or metastatic pancreatic carcinomas.

\begin{tabular}{|c|c|c|c|c|c|}
\hline Trial & Year & Country/Region & $\mathbf{N}$ & Regimens & Outcomes \\
\hline Burris [47] & 1997 & $\begin{array}{l}\text { USA and } \\
\text { Canada }\end{array}$ & 126 & $\begin{array}{c}\text { Gemcitabine }(n=63) \text { vs. } \\
\text { 5-FU }(n=63)\end{array}$ & $\begin{array}{c}\text { mOS } 5.65 \text { mo vs. } 4.41 \text { mo ( } p= \\
0.0025) ; 1 \text {-year OS } 18 \% \text { vs. } 2 \% ; \\
\text { mPFS } 2.33 \text { mo vs. } 0.92 \text { mo ( } p= \\
0.0002) ; 1 \text {-year PFS } 9 \% \text { vs. } 5 \% \text {; } \\
\text { clinical benefit response } 23.8 \% \text { vs. } \\
4.8(p=0.0022)\end{array}$ \\
\hline Huguier [48] & 2001 & France & 45 & $\begin{array}{c}5 \text {-FU }+ \text { leucovorin }+ \\
\text { cisplatin }(n=22) \text { vs. best } \\
\text { supportive care }(n=23)\end{array}$ & mOS 8.6 mo vs. $7.0 \mathrm{mo}$ \\
\hline Ducreux [49] & 2002 & France & 207 & $\begin{array}{l}5-\mathrm{FU}+\text { cisplatin }(n=104) \mathrm{vs} . \\
5-\mathrm{FU}(n=103)\end{array}$ & $\begin{array}{c}\text { response } 12 \% \text { vs. } 0 \%(p<0.01) \\
1 \text {-year OS } 17 \% \text { vs. } 9 \%(p=0.10) \\
1 \text {-year PFS } 10 \% \text { vs. } 0 \%(p=0.0001)\end{array}$ \\
\hline Colucci [50] & 2002 & Italy & 107 & $\begin{array}{l}\text { Gemcitabine + cisplatin }(n= \\
53) \text { vs. gemcitabine }(n=54)\end{array}$ & $\begin{array}{c}\text { mOS } 30 \text { weeks vs. } 20 \text { weeks }(p= \\
0.43) ; \text { response } 26.4 \% \text { vs. } 9.2 \% \text { ( } p= \\
0.02) ; \text { mTTP (time to progression) } 20 \\
\text { weeks vs. } 8 \text { weeks }(p=0.048)\end{array}$ \\
\hline $\begin{array}{l}\text { Scheithauer } \\
\text { [51] }\end{array}$ & 2003 & Austria & 83 & $\begin{array}{c}\text { Gemcitabine }+ \text { capecitabine } \\
(2500 \mathrm{mg} / \mathrm{m} 2 \text { qd } 1 / 2 \text { weeks }) \\
(n=41) \text { vs. gemcitabine } \\
\text { (high-dose intense) }(n=42)\end{array}$ & $\begin{array}{c}\text { mOS } 9.5 \text { mo vs. } 8.2 \text { mo; } 1 \text {-year OS } \\
31.8 \% \text { vs. } 37.2 \% \text {; mPFS } 5.1 \text { mo vs. } \\
4.0 \text { mo; response } 17 \% \text { vs. } 14 \% \text {; } \\
\text { clinical benefit } 48.4 \% \text { vs. } 33 \% \text {; } \\
\text { values not reported }\end{array}$ \\
\hline Tempero [52] & 2003 & $\begin{array}{l}\text { USA and } \\
\text { Netherlands }\end{array}$ & 92 & $\begin{array}{l}\text { dose-intense gemcitabine }(n \\
=49) \text { vs. fixed dose rate } \\
\text { gemcitabine }(n=43)\end{array}$ & $\begin{array}{l}\text { mOS } 5.0 \text { mo vs. } 8.0 \text { mo }(p=0.013) ; \\
\text { 1-year OS } 9 \% \text { vs. } 28.8 \%(p=0.014) ; \\
\text { 2-year OS } 2.2 \% \text { vs. } 18.3 \%(p=0.007) ; \\
\text { mPFS } 1.9 \text { mo vs. } 3.4 \text { mo }(p=0.68) \\
\text { mTTF (time to treatment failure) } 1.8 \\
\text { mo vs. } 2.1 \text { mo }(p=0.09)\end{array}$ \\
\hline Ducreux [53] & 2004 & France & 63 & $\begin{array}{l}\text { 5-FU + oxaliplatin }(n=31) \\
\text { vs. } 5 \text {-FU }(n=15) \text { vs. } \\
\quad \text { oxaliplatin }(n=17)\end{array}$ & $\begin{array}{l}\text { mOS } 9.0 \text { mo vs. } 2.4 \text { mo vs. } 3.4 \text { mo; } \\
\text { mPFS } 4.2 \text { mo vs. } 1.5 \text { mo vs. } 2.0 \text { mo; } \\
\text { response } 10 \% \text { vs. } 0 \% \text { vs. } 0 \% \text {; stable } \\
48 \% \text { vs. } 20 \% \text { vs. } 12 \%\end{array}$ \\
\hline $\begin{array}{l}\text { Louvet } \\
\text { (GERCOR } \\
\text { GISCAD) [54] }\end{array}$ & 2005 & $\begin{array}{l}\text { France and } \\
\text { Italy }\end{array}$ & 326 & $\begin{array}{l}\text { Gemcitabine + oxaliplatin }(n \\
=157) \text { vs. gemcitabine } \\
(n=156)\end{array}$ & $\begin{array}{c}\text { mOS } 9.0 \text { mo vs. } 7.1 \mathrm{mo}(p=0.13) \\
\text { 1-year OS } 34.7 \% \text { vs. } 27.8 \%(p=0.22) \\
\text { mPFS } 5.8 \text { mo vs. } 3.7 \mathrm{mo}(p=0.04) \\
\text { response } 26.8 \% \text { vs. } 17.3 \%(p=0.04) \\
\text { clinical benefit } 38.2 \% \text { vs. } 26.9 \% \\
(p=0.03)\end{array}$ \\
\hline $\begin{array}{l}\text { Heinemann } \\
\text { [55] }\end{array}$ & 2006 & Germany & 195 & $\begin{array}{l}\text { Gemcitabine + cisplatin }(n= \\
98) \text { vs. gemcitabine }(n=97)\end{array}$ & $\begin{array}{c}\text { mOS } 7.5 \text { mo vs. } 6.0 \text { mo }(p=0.15) \\
\text { 1-year OS } 25.3 \% \text { vs. } 24.7 \%(p=0.21) ; \\
\text { mPFS } 5.3 \text { mo vs. } 3.1 \text { mo }(p=0.053) ; \\
\text { response } 10.2 \% \text { vs. } 8.2 \% \text { ns; stable } \\
\quad 60.2 \% \text { vs. } 40.2 \%(p<0.001)\end{array}$ \\
\hline $\begin{array}{l}\text { Moore (NCIC } \\
\text { CTG PA.3) [56] }\end{array}$ & 2007 & International & 569 & $\begin{array}{l}\text { Gemcitabine plus erlotinib } \\
(n=285) \text { vs. gemcitabine } \\
\text { plus placebo }(n=284)\end{array}$ & $\begin{array}{c}\text { mOS } 6.24 \text { mo vs. } 5.91 \mathrm{mo}(p=0.038) \\
1 \text {-year OS } 23 \% \text { vs. } 17 \%(p=0.023) ; \\
\text { mPFS } 3.75 \text { mo vs. } 3.55 \text { mo }(p= \\
0.004) ; \text { control } 57.5 \% \text { vs. } 49.2 \% \\
(p=0.07)\end{array}$ \\
\hline $\begin{array}{l}\text { Herrmann [57] } \\
\quad \text { (SAKK } \\
\text { 44/00-CECOG/ } \\
\text { PAN.1.3.001) }\end{array}$ & 2007 & Europe & 319 & $\begin{array}{c}\text { Gemcitabine }+ \text { capecitabine } \\
(650 \mathrm{mg} / \mathrm{m} 2 \text { bid po } 2 / 3 \\
\text { weeks })(n=160) \text { vs. } \\
\text { gemcitabine (standard dose) } \\
\qquad(n=159)\end{array}$ & $\begin{array}{c}\text { mOS } 8.4 \text { mo vs. } 7.2 \text { mo }(p=0.234) \\
1 \text {-year OS } 32 \% \text { vs. } 30 \% \text {; mPFS } 4.3 \\
\text { mo vs. } 3.9 \text { mo }(p=0.103) \text {; response } \\
10.0 \% \text { vs. } 7.8 \% \text {; clinical benefit } 19 \% \\
\text { vs. } 20 \%\end{array}$ \\
\hline
\end{tabular}


Table 3. Cont.

\begin{tabular}{|c|c|c|c|c|c|}
\hline Trial & Year & Country/Region & $\mathbf{N}$ & Regimens & Outcomes \\
\hline Boeck [58] & 2008 & Germany & 190 & $\begin{array}{c}\text { Capecitabine plus } \\
\text { oxaliplatin }(n=61) \text { vs. } \\
\text { capecitabine plus } \\
\text { gemcitabine }(n=64) \text { vs. } \\
\text { gemcitabine plus oxaliplatin } \\
\quad(n=63)\end{array}$ & $\begin{array}{l}\text { mOS } 8.1 \text { mo vs. } 9.0 \text { mo vs. } 6.9 \text { mo ( } p \\
=0.56) ; 1 \text {-year OS } 29 \% \text { vs. } 33 \% \text { vs. } \\
22 \% \text {; mPFS } 4.2 \text { mo vs. } 5.7 \text { mo vs. } 3.9 \\
\text { mo ( } p=0.67) ; 1-\text {-year PFS } 8 \% \text { vs. } \\
14 \% \text { vs. } 8 \% ; \text { response } 13 \% \text { vs. } 25 \% \\
\text { vs. } 13 \%(p=0.13)\end{array}$ \\
\hline $\begin{array}{l}\text { Cunningham } \\
\text { [59] }\end{array}$ & 2009 & UK & 533 & $\begin{array}{l}\text { Gemcitabine + capecitabine } \\
(830 \mathrm{mg} / \mathrm{m} 2 \text { bid po } 3 / 4 \\
\text { weeks })(n=267) \text { vs. } \\
\text { gemcitabine (standard dose }) \\
\qquad(n=266)\end{array}$ & $\begin{array}{c}\text { mOS } 7.1 \text { mo vs. } 6.2 \text { mo }(p=0.08) \\
1 \text {-year OS } 24.3 \% \text { vs. } 22.0 \% \text {; mPFS } \\
5.3 \text { mo vs. } 3.8 \text { mo }(p=0.004) ; 1 \text {-year } \\
\text { PFS } 13.9 \% \text { vs. } 8.4 \% \text {; response } 19.1 \% \\
\text { vs. } 12.4 \%(p=0.03)\end{array}$ \\
\hline $\begin{array}{l}\text { Poplin (E6201) } \\
\text { [60] }\end{array}$ & 2009 & USA & 824 & $\begin{array}{l}\text { Gemcitabine }(n=275) \text { vs. } \\
\text { fixed dose rate gemcitabine } \\
(n=277) \text { vs. gemcitabine } \\
\text { plus oxaliplatin }(n=272)\end{array}$ & $\begin{array}{c}\text { mOS } 4.9 \text { mo vs. } 6.2 \text { mo vs. } 5.7 \text { mo; } \\
\text { 1-year OS } 16 \% \text { vs. } 22 \% \text { vs. } 21 \% \text {; } \\
2 \text {-year OS } 4 \% \text { vs. } 6 \% \text { vs. } 6 \% ; \mathrm{mPFS} \\
2.6 \text { mo vs. } 3.5 \text { mo vs. } 2.7 \mathrm{mo}\end{array}$ \\
\hline $\begin{array}{l}\text { Kulke (CALGB } \\
\text { 89904) [61] }\end{array}$ & 2009 & USA & 245 & $\begin{array}{l}\text { Gemcitabine plus cisplatin } \\
(n=62) \text { vs. fixed dose rate } \\
\text { gemcitabine }(n=58) \text { vs. } \\
\text { gemcitabine plus docetaxel } \\
(n=65) \text { vs. gemcitabine plus } \\
\text { irinotecan }(n=60)\end{array}$ & $\begin{array}{c}\text { mOS } 6.7 \text { mo vs. } 6.4 \text { mo vs. } 6.4 \text { mo } \\
\text { vs. } 7.1 \text { mo; mTTP } 4.5 \text { mo vs. } 3.3 \text { mo } \\
\text { vs. } 4.1 \text { mo vs. } 4.0 \text { mo; response } 13 \% \\
\text { vs. } 14 \% \text { vs. } 12 \% \text { vs. } 14 \%\end{array}$ \\
\hline $\begin{array}{l}\text { Colucci (GIP-1) } \\
\text { [62] }\end{array}$ & 2010 & Italy & 400 & $\begin{array}{c}\text { Gemcitabine }+ \text { cisplatin }(n= \\
\text { 201) vs. gemcitabine }(n= \\
\text { 199) }\end{array}$ & $\begin{array}{c}\text { mOS } 7.2 \text { mo vs. } 8.3 \text { mo }(p=0.38) \\
1 \text {-year OS } 30.7 \% \text { vs. } 34.0 \% \text {; mPFS } \\
3.8 \text { mo vs. } 3.9 \text { mo }(p=0.80) ; 1 \text {-year } \\
\text { PFS } 14.5 \% \text { vs. } 12.8 \% \text {; response } \\
12.9 \% \text { vs. } 10.1 \% \text { ( } p=0.37) ; \text { clinical } \\
\text { benefit } 15.1 \% \text { vs. } 23.0 \%(p=0.057)\end{array}$ \\
\hline $\begin{array}{c}\text { Dahan (FFCD } \\
\text { 0301) [63] }\end{array}$ & 2010 & France & 202 & $\begin{array}{l}\text { 5-FU/folinic acid/cisplatin } \\
\text { followed by gemcitabine }(n \\
=102) \text { vs. gemcitabine } \\
\text { followed by } 5 \text {-FU/folinic } \\
\text { acid/cisplatin }(n=100)\end{array}$ & $\begin{array}{l}\text { mOS } 6.7 \text { mo vs. } 8.03 \text { mo }(p=0.83) \\
\text { mPFS } 3.4 \text { mo vs. } 3.5 \text { mo }(p=0.67) \\
\text { response } 15 \% \text { vs. } 19 \%\end{array}$ \\
\hline $\begin{array}{l}\text { PRODIGE 4/ } \\
\text { ACCORD } 11 \\
\quad[64]\end{array}$ & 2011 & France & 342 & $\begin{array}{l}\text { FOLFIRINOX }(n=171) \text { vs. } \\
\text { gemcitabine }(n=171)\end{array}$ & $\begin{array}{c}\text { mOS } 11.1 \text { mo vs. } 6.8 \text { mo }(\mathrm{P}<0.001) \\
1 \text {-year OS } 48.4 \% \text { vs. } 20.6 \% \text {; mPFS } \\
6.4 \text { mo vs. } 3.3 \text { mo }(p<0.001) ; 1 \text {-year } \\
\text { PFS } 12.1 \% \text { vs. } 3.5 \% \text {; response } 31.6 \% \\
\text { vs. } 9.4 \%(p<0.001) ; 6 \text {-month } \\
\text { degradation QoL } 31 \% \text { vs. } 66 \% \text { ( } p< \\
0.001)\end{array}$ \\
\hline $\begin{array}{c}\text { Ozaka } \\
\text { (JACCRO } \\
\text { PC-01) [65] }\end{array}$ & 2012 & Japan & 112 & $\begin{array}{l}\text { Gemcitabine plus S- } 1(n= \\
53) \text { vs. gemcitabine }(n=59)\end{array}$ & $\begin{array}{c}\text { mOS } 13.7 \text { mo vs. } 8.0 \text { mo }(p=0.035) \\
1 \text {-year OS } 55.9 \% \text { vs. } 29.0 \% ; \text { mPFS } \\
6.15 \text { mo vs. } 3.78 \text { mo }(p=0.0007) ; \\
\text { response } 28.3 \% \text { vs. } 6.8 \%(p=0.005)\end{array}$ \\
\hline $\begin{array}{c}\text { Nakai } \\
\text { (GEMSAP) [66] }\end{array}$ & 2012 & Japan & 106 & $\begin{array}{l}\text { Gemcitabine plus S- } 1(n= \\
53) \text { vs. gemcitabine }(n=53)\end{array}$ & $\begin{array}{c}\text { mOS } 13.5 \text { mo vs. } 8.8 \text { mo }(p=0.104) \\
\text { 1-year OS } 52.8 \% \text { vs. } 30.2 \%(p= \\
0.031) ; \text { mPFS } 5.4 \text { mo vs. } 3.6 \text { mo }(p= \\
0.036) ; \text { response } 18.9 \% \text { vs. } 9.4 \%(p= \\
0.265)\end{array}$ \\
\hline Chao [67] & 2013 & Taiwan & 46 & $\begin{array}{l}\text { Gemcitabine + ciaplatin }(n= \\
21) \text { vs. gemcitabine }(n=25)\end{array}$ & $\begin{array}{c}\text { mOS } 7.9 \text { mo vs. } 7.7 \text { mo }(p=0.752) \\
1 \text {-year OS } 9.5 \% \text { vs. } 12 \% \text {; mTTP } 3.6 \\
\text { mo vs. } 4.6 \text { mo }(p=0.857) \text {; partial } \\
\text { response } 4.8 \% \text { vs. } 8 \%(p=1) ; \text { clinical } \\
\text { benefit } 29 \% \text { vs. } 36 \%(p=0.592)\end{array}$ \\
\hline $\begin{array}{l}\text { Von Hoff and } \\
\text { Goldstein } \\
\text { (MPACT) [68] }\end{array}$ & $\begin{array}{l}2013 \\
\text { and } \\
2015\end{array}$ & $\begin{array}{l}\text { International } 11 \\
\text { countries }\end{array}$ & 861 & $\begin{array}{c}\text { Gemcitabine }+ \\
\text { nab-paclitaxel }(n=431) \text { vs. } \\
\text { gemcitabine }(n=430)\end{array}$ & $\begin{array}{c}\text { mOS } 8.7 \text { mo vs. } 6.6 \text { mo }(p<0.001) ; \\
1 \text {-year OS } 35 \% \text { vs. } 22 \%(p<0.001) ; \\
\text { 2-year OS } 10 \% \text { vs. } 5 \% \text {; } 3 \text {-year OS } 4 \% \\
\text { vs. } 0 \% \text {; mPFS } 5.5 \text { mo vs. } 3.7 \text { mo }(p< \\
0.001) ; 1 \text {-year PFS } 16 \% \text { vs. } 9 \% ; \\
\text { response } 23 \% \text { vs. } 7 \%(p<0.001) ; \\
\text { mTTF } 5.1 \text { mo vs. } 3.6 \text { mo }(p<0.001)\end{array}$ \\
\hline
\end{tabular}


Table 3. Cont.

\begin{tabular}{|c|c|c|c|c|c|}
\hline Trial & Year & Country/Region & $\mathbf{N}$ & Regimens & Outcomes \\
\hline $\begin{array}{c}\text { Ueno and } \\
\text { Okusaka } \\
\text { (GEST) }[69,70]\end{array}$ & $\begin{array}{c}2013 \\
\text { and } \\
2017\end{array}$ & $\begin{array}{c}\text { Japan and } \\
\text { Taiwan }\end{array}$ & 834 & $\begin{array}{l}\text { Gemcitabine plus S-1 }(n= \\
275) \text { vs. S-1 }(n=280) \text { vs. } \\
\text { gemcitabine }(n=277)\end{array}$ & $\begin{array}{l}\text { mOS } 9.9 \text { mo vs. } 9.7 \text { mo vs. } 8.8 \text { mo; } \\
\text { 1-year OS } 40.7 \% \text { vs. } 38.7 \% \text { vs. } 35.4 \% \text {; } \\
\text { 2-year OS } 14.5 \% \text { vs. } 12.7 \% \text { vs. } 9.2 \% \text {; } \\
\text { mPFS } 5.7 \text { mo vs. } 3.8 \text { mo vs. } 4.1 \text { mo; } \\
\text { 1-year PFS } 20.3 \% \text { vs. } 7.2 \% \text { vs. } 9.1 \% \text {; } \\
\text { response } 29.3 \% \text { vs. } 21.0 \% \text { vs. } 13.3 \%\end{array}$ \\
\hline Sudo [71] & 2014 & Japan & 101 & $\begin{array}{l}\text { Gemcitabine plus S-1 }(n= \\
51) \text { vs. gemcitabine }(n=50)\end{array}$ & $\begin{array}{l}\text { mOS } 8.6 \text { mo vs. } 8.6 \text { mo }(p=0.714) \\
\text { mPFS } 5.3 \text { mo vs. } 3.8 \text { mo }(p=0.039) \\
\text { response } 21.6 \% \text { vs. } 6 \%(p=0.048)\end{array}$ \\
\hline Petrioli [72] & 2015 & Italy & 67 & $\begin{array}{l}\text { Gemcitabine }+ \text { capecitabine } \\
+ \text { oxaliplatin }(n=34) \text { vs. } \\
\text { gemcitabine }(n=33)\end{array}$ & $\begin{array}{l}\text { mOS } 11.9 \text { mo vs. } 7.1 \text { mo }(p<0.001) \\
\text { mPFS } 6.8 \text { mo vs. } 3.7 \text { mo }(p<0.001) \\
\text { 4-month control } 79.4 \% \text { vs. } 45.4 \% \\
(p=0.08)\end{array}$ \\
\hline Wang [73] & 2015 & Taiwan & 88 & $\begin{array}{l}\text { Gemcitabine plus erlotinib } \\
(n=44) \text { vs. gemcitabine } \\
(n=44)\end{array}$ & $\begin{array}{l}\text { mOS } 7.2 \text { mo vs. } 4.4 \text { mo }(p<0.001) \\
\text { mPFS } 3.8 \text { mo vs. } 2.4 \text { mo }(p<0.001) \\
\text { control } 64 \% \text { vs. } 25 \%(p<0.001)\end{array}$ \\
\hline Lee [74] & 2017 & Korea & 214 & $\begin{array}{l}\text { Gemcitabine }+ \text { capecitabine } \\
\quad(830 \mathrm{mg} / \mathrm{m} 2 \text { bid po } 3 / 4 \\
\text { weeks })(n=103) \text { vs. } \\
\text { gemcitabine (standard dose }) \\
\qquad(n=101)\end{array}$ & $\begin{array}{l}\text { mOS } 10.3 \text { mo vs. } 7.5 \text { mo }(p=0.06) \\
\text { mPFS } 6.2 \text { mo vs. } 5.3 \text { mo }(p=0.08) ; \\
\text { response } 43.7 \% \text { vs. } 17.6 \%(p=0.001)\end{array}$ \\
\hline
\end{tabular}

For locally advanced non-metastatic pancreatic cancer, the additional role of radiotherapy has been studied, but the survival benefits of chemoradiotherapy (CRT) are still undetermined. There is currently very limited evidence from randomized clinical trials supporting the use of CRT in this setting. In a systematic review, Huguet and colleagues indicated that CRT increased overall survival when compared with best supportive care or exclusive radiotherapy, but it was more toxic [76]. The advantage of CRT over exclusive chemotherapy is inconsistent (Table 4) [77-83]. Induction chemotherapy may select patients with locally advanced pancreatic cancer for optimal benefit from CRT by excluding patients with rapid progressive disease. Two retrospective studies indicated significantly improved survival benefit with the use of induction chemotherapy before continuing with CRT, as compared with upfront CRT or with exclusive chemotherapy $[84,85]$. A recent meta-analysis showed that induction chemotherapy followed by consolidation CRT did not significantly improve survival in patients with locally advanced pancreatic cancer as compared to chemotherapy alone; however, the survival benefit of this treatment strategy over chemotherapy alone was noted only when induction chemotherapy lasted for at least 3 months [86].

In patients with locally advanced unresectable tumor at primary diagnosis, resectability should be reassessed after first treatment. Patients presenting significant response to chemotherapy and/or CRT should undergo surgery if $\mathrm{R} 0$ resection seems to be achievable according to the latest imaging as this significantly improves their prognosis (preliminary data from CONKO 007 trial).

When first-line treatment fails, second-line therapy is increasingly considered in patients with good performance status, as it is suggested in association with improved survival $[87,88]$. There is a paucity of clinical evidence supporting any optimal second-line regimens for patients with advanced pancreatic cancer. For those who have received prior gemcitabine-based therapy, fluoropyrimidine-based chemotherapy regimens are generally accepted as the second-line options. On the other hand, gemcitabine-based therapy could be used to those previously treated with fluoropyrimidine-based therapy. Chemoradiation could be considered in patients with locally advanced disease, but palliative radiotherapy may be administered to patients with locally metastatic disease and poor performance status. 
Table 4. Summary of randomized controlled trials concerning chemoradiation in patients with locally advanced pancreatic carcinomas.

\begin{tabular}{|c|c|c|c|c|c|}
\hline Trial & Year & Country/Region & $\mathbf{N}$ & Regimens & Outcomes \\
\hline $\begin{array}{l}\text { Chauffert [77] } \\
\quad(2000-01 \\
\text { FFCD/SFRO) }\end{array}$ & 2008 & France & 119 & $\begin{array}{l}\text { CRT (60 Gy, 5-FU/cisplatin) plus } \\
\text { maintenance gemcitabine } \\
\text { chemotherapy }(n=59) \text { vs. } \\
\text { gemcitabine chemotherapy } \\
(n=60)\end{array}$ & $\begin{array}{c}\text { mOS } 8.6 \text { mo vs. } 13 \text { mo } \\
(p=0.03) ; 1 \text {-year OS } 32 \% \text { vs. } \\
53 \% ; 1 \text {-year PFS } 14 \% \text { vs. } 32 \%\end{array}$ \\
\hline Loehrer [78] & 2011 & USA & 74 & $\begin{array}{l}\text { CRT (50.4 Gy, gemcitabine) } \\
(n=34) \text { vs. gemcitabine } \\
\text { chemotherapy }(n=37)\end{array}$ & $\begin{array}{c}\text { mOS } 11.1 \text { mo vs. } 9.2 \text { mo } \\
(p=0.017) ; \text { mPFS } 6.0 \text { mo vs. } \\
6.7 \text { mo; response } 6 \% \text { vs. } 5 \% \\
\text { stable } 68 \% \text { vs. } 35 \%\end{array}$ \\
\hline \multirow[t]{2}{*}{$\begin{array}{c}\text { Hammel } \\
\text { (LAP-07) [79] }\end{array}$} & 2016 & International & 449 & $\begin{array}{c}\text { gemcitabine }(n=223) \text { vs. } \\
\text { gemcitabine + erlotinib }(n=219)\end{array}$ & $\begin{array}{l}\text { From first randomization, } \\
\text { mOS } 13.6 \text { mo vs. } 11.9 \text { mo }(p= \\
0.09) ; \text { mPFS } 7.8 \text { mo vs. } 6.5 \text { mo } \\
\quad(p=0.26)\end{array}$ \\
\hline & & & 269 & $\begin{array}{c}\text { chemotherapy same as previously } \\
\text { for } 2 \text { mo }(n=136) \text { vs. CRT ( } 54 \text { Gy, } \\
\text { capecitabine) }(n=133)\end{array}$ & $\begin{array}{l}\text { From first randomization, } \\
\text { mOS } 16.5 \text { mo vs. } 15.2 \mathrm{mo}(p= \\
0.83) ; \text { mPFS } 8.4 \text { mo vs. } 9.9 \text { mo } \\
\text { ( } p=0.06) ; \text { local progression } \\
46 \% \text { vs. } 32 \%(p=0.03)\end{array}$ \\
\hline Li [80] & 2003 & Taiwan & 34 & $\begin{array}{l}\text { CRT (50.4 61.2 Gy, gemcitabine) } \\
(n=18) \text { vs. CRT (50.4 61.2 Gy, } \\
\text { 5-FU) }(n=16), \text { both followed by } \\
\text { gemcitabine chemotherapy }\end{array}$ & $\begin{array}{c}\text { mOS } 14.5 \text { mo vs. } 6.7 \text { mo }(p= \\
0.027) ; 1-y \text { OS } 56 \% \text { vs. } 31 \% ; \\
2 \text {-y OS } 15 \% \text { vs. } 0 \% \text {; mTP } 7.1 \\
\text { mo vs. } 2.7 \text { mo ( } p=0.019) ; \\
\text { response } 50 \% \text { vs. } 12.5 \% \\
(p=0.005)\end{array}$ \\
\hline Wilkowski [81] & 2009 & Germany & 95 & $\begin{array}{c}\text { CRT (50 Gy, 5-FU) ( } n=30) \text { vs. } \\
\text { CRT ( } 50 \text { Gy, gemcitabine/cisplatin) } \\
(n=32) \text { vs. CRT ( } 50 \text { Gy, } \\
\text { gemcitabine/cisplatin) followed } \\
\text { by gemcitabine/cisplatin } \\
\text { chemotherapy }(n=31)\end{array}$ & $\begin{array}{l}\text { mOS } 9.6 \text { mo vs. } 9.3 \text { mo vs. } 7.3 \\
\text { mo ( } p=0.61) ; 9-\text { mo OS } 58 \% \text { vs. } \\
52 \% \text { vs. } 45 \% \text {; mPFS } 4.0 \text { mo vs. } \\
5.6 \text { mo vs. } 6.0 \text { mo ( } p=0.21) ; \\
\text { response } 19 \% \text { vs. } 22 \% \text { vs. } 13 \%\end{array}$ \\
\hline $\begin{array}{c}\text { Mukherjee and } \\
\text { Hurt }[82,83]\end{array}$ & $\begin{array}{l}2013 \\
\text { and } \\
2017\end{array}$ & UK & 114 & $\begin{array}{l}\text { Induction chemotherapy with } \\
\text { gemcitabine and capecitabine for } \\
12 \text { weeks, if no tumor progression, } \\
\text { then chemotherapy with } \\
\text { gemcitabine and capecitabine for } \\
\text { another cycle; then CRT ( } 50.4 \text { Gy, } \\
\text { capecitabine) }(n=36) \text { vs. CRT } \\
\text { (50.4 Gy, gemcitabine) }(n=38)\end{array}$ & $\begin{array}{c}\text { mOS } 17.6 \text { mo vs. } 14.6 \text { mo }(p= \\
0.185) ; 1-y e a r \text { OS } 79.2 \% \text { vs. } \\
64.2 \% \text {; mPFS } 12.0 \text { mo vs. } 10.4 \\
\text { mo }(p=0.120) ; 9 \text {-mo PFS } \\
62.9 \% \text { vs. } 51.4 \% \text {; response ( } 26 \\
\text { weeks) } 23 \% \text { vs. } 19 \%\end{array}$ \\
\hline
\end{tabular}

\subsection{Palliative Care}

A considerable proportion of patients with pancreatic cancer require palliative interventions to relieve symptoms and ensure optimal quality of life. Biliary obstruction is one of the most common severe circumstances in patients with pancreatic cancer. Placement of self-expanding metal stents is the preferred method to relieve biliary obstruction in patients with unresectable disease, as it is associated with lower rates of, and longer time to, recurrent biliary obstruction as compared to plastic stents, resulting in less cholangitis $[89,90]$. Placing the stents endoscopically is preferable as it is safer than percutaneous insertion; however, percutaneous biliary drainage is an alternative option when an endoscopic stent cannot be placed. Another option is surgical biliary bypass, which is considered when the cancer is found unresectable during attempted resection, as it provides durable palliation and potentially avoids additional stent-insertion procedure [91]. For patients with gastric outlet obstruction, endoscopically placed enteral stent is preferred for patients with a short life expectancy or poor performance status, while gastrojejunostomy is considered more effective for patients with longer life expectancy and favorable prognosis [92]. As the quality of life is markedly hampered if gastric outlet obstruction occurs, prophylactic gastrojejunostomy could be considered for those at risk 
of developing symptomatic gastric outlet obstruction but otherwise fit and with a relatively good prognosis [93].

Most patients with locally advanced or metastatic pancreatic cancer develop cancer-related pain. The mainstay of pain management in these patients is administration of analgesics. However, for those whose analgesic control shows inadequate or with undesirable side effects, EUS- or image-guided celiac plexus neurolysis could significantly improve pain relief [94]. In selected patients with severe local back pain refractory to analgesia, palliative radiation might be considered to ameliorate pain [95]. Except pain, malnutrition is also prevalent in patients with pancreatic cancer. A proper nutritional evaluation should be performed, and oral pancreatic enzyme should be administered in patients with both unresectable and resected pancreatic cancer [96]. Additionally, the risk of developing venous thromboembolism is substantially increased in patients with pancreatic cancer. Low molecular weight heparin is preferably administered, as randomized clinical trials indicated significantly decreased incidence of venous thromboembolism associated with intake of low molecular weight heparins $[97,98]$.

\subsection{Conclusion/Future Directions}

Even with the latest efforts for novel therapeutic strategies, especially new chemo(radio)therapy regimens in (neo)adjuvant settings and improved surgical options, the clinical outcome of patients with pancreatic cancer remains disappointing. Clinically, we anticipate a higher amount of neoadjuvant therapeutic approaches for patients with non-metastatic pancreatic cancer in the near future; however, a better understanding of the underlying molecular mechanisms of this disease is of central importance to design new therapeutic strategies for all patients (Figure 1b). For molecular testing of pancreatic cancer, an individualized therapeutic concept for each patient might be available, thus leading to a better prediction of the patient's prognosis, a better prediction of the effectiveness of the available chemotherapeutics, and finally improvement in the patient's outcome.

\section{Immunotherapy and Other State-of-the-Art Molecular Options}

Knowledge of the molecular aspects is becoming increasingly important in the therapy and prognosis of patients with ductal pancreatic carcinoma. The understanding of pancreatic carcinoma at the molecular level is a complex interplay of various factors (Figure 2): In patients with pancreatic ductal adenocarcinoma (PDAC) the tumor microenvironment, consisting of cellular and stromal components, plays an important role and influence prognosis leading to development of tumor vaccination as a potential future treatment option. The influence of genes plays a decisive role as well. The most common genes in pancreatic carcinoma are KRAS, TP53, CDKN2A, and SMAD4/TGFBR1/2. In addition to the immunological and genetic components, epigenetic modifications and molecular subtypes have a significant influence on PDAC. Promising findings for diagnostic and treatment are expected from liquid biopsy.

\subsection{Immunotherapy}

Pancreatic cancers are characterized by an immunosuppressive microenvironment due to the dysfunction of immune effector CD8+ T cells and Natural killer (NK) cells, which is a result of the involvement of multiple types of immune cells, including cancer-associated fibroblasts, regulatory $\mathrm{T}$ cells, myeloid-derived suppressor cells, tumor-associated macrophages, and tumor-infiltrating lymphocytes [99]. The immune suppression occurs through both the expression of tolerance-inducing cell surface molecules (PD-1, CTLA-4, and CD40) and the secretion of immunosuppressing cytokines (IL-10, TGF- $\beta$ ) [100]. The function of the immune system is therefore converted from anti-cancer immunity to a supportive microenvironment that fosters the growth and invasion of the tumor and helps the tumor escape from host immune surveillance. Thus, strategies could be exploited to disrupt this immunosuppressive network and promote the tumoricidal activity of these immune effector cells to potentially improve the outcome of the patients [100,101]. 


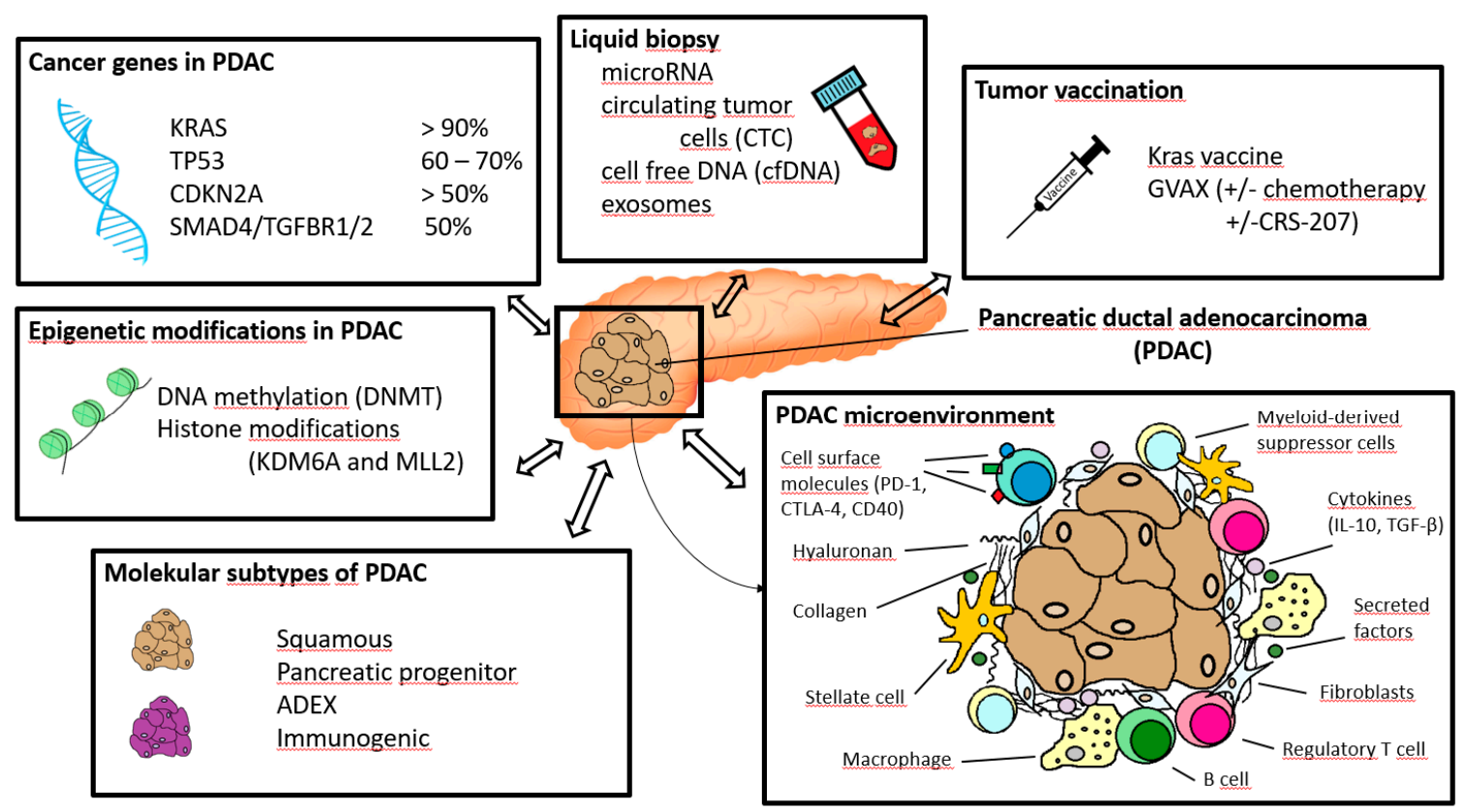

Figure 2. Overview of important aspects in immunotherapy and molecular pathology of pancreatic ductal adenocarcinoma.

$\mathrm{T}$ cells are activated when antigen presenting cells (APCs) present antigen peptides on both major histocompatibility complex (MHC) class I (for CD8+ T cells) and class II (for CD4+ T helper cells) molecules to the T cell receptor (TCR) [100]. Effective activation of T cells also requires additional ligand binding of co-stimulatory receptors, such as CD40, which is a member of the tumor necrosis factor (TNF) receptor family presented on APCs such as tumor-associated macrophages. CD40 on the surface of APCs could bind with the CD40 ligand expressed on activated CD4+ T cells, thus forming a stimulatory loop. CD40 and its ligand (CD154) are both expressed in a subset of pancreatic patients, and the high expression of CD40 ligand is associated with significantly better prognosis than others [102]. The activation of CD40 with a CD40 agonist in combination with gemcitabine is indicated to promote accumulation of tumoricidal macrophages within the tumors in the KPC mouse model, which resulted in stromal collapse and tumor regression in the KPC mouse model and some advanced-stage pancreatic cancer patients $[103,104]$. On the other hand, inhibitory receptors such as programed cell death-1 (PD-1) and cytotoxic T-lymphocyte associated protein 4 (CTLA-4) expressed on the surface of T cells inhibit $\mathrm{T}$ cell activation upon binding to their ligands. PD-1 is an immune checkpoint receptor expressed on activated T cells; whereas PD-1 ligands (PD-L1 and PD-L2) could be expressed on stroma and cancer cells. The binding of PD-1 ligands to PD-1 inactivates T cell responses to the cells presenting these ligands, i.e., the pancreatic cancer cells and stroma in this situation, facilitating the cancer to escape from the host's immune surveillance [101]. PD-L1 expression, but not PD-L2, is correlated to significantly poorer survival than the ligand-negative patients, and it is inversely correlated with tumor-infiltrating lymphocytes within the tumor, particularly CD8+ T cells [105]. In vivo experiments suggested that blockade of PD-1 signaling with anti-PD-L1 or anti-PD-1 monoclonal antibody promoted CD8 + T cells infiltration into the tumor, induced local immune activation, and finally resulted in substantial anti-tumor effects in a murine pancreatic cancer model [105]. However, the effect of PD-L1 inhibitors in treating patients with pancreatic cancer was low [106]. A preclinical study using the KPC mouse model suggested that depletion of fibroblast-associated protein (FAP)-positive cancer-associated fibroblasts (CAFs) sensitized the tumor to the treatment with anti-PD-L1 monoclonal antibody [107]. FAP-positive CAFs in tumor stroma are the major source of C-X-C motif ligand 12 (CXCL12) in the tumor, and inhibition of its receptor, the C-X-C motif receptor 4 (CXCR4), induced T cell accumulation in the tumor and acted synergistically with anti-PD-L1 in diminishing cancer cells [107]. In addition, 
PD-L1 inhibitor and gemcitabine chemotherapy also showed synergistic effect on pancreatic cancer in preclinical mouse model [105]. CTLA-4 is another inhibitory checkpoint protein expressed on regulatory T cells and exhausted CD8+ T cells. In a transgenic mouse model, depletion of myofibroblasts induced an immunosuppressive microenvironment, as characterized by decreased overall immune infiltration but increased number of regulatory $\mathrm{T}$ cells, which then accelerated the progression of pancreatic cancer leading to reduced survival [108]. The use of anti-CTLA-4 monoclonal antibody reversed the accelerated disease progression and prolonged survival in this CAFs-depleted mouse model. Although single agent Ipilimumab (an anti-CTLA-4 monoclonal antibody) immunotherapy was shown in a phase 2 study ineffective in the treatment of advanced pancreatic cancer, it might be considered when combination chemotherapy with nab-paclitaxel and gemcitabine is applied, as this regimen decreased the number of CAFs in the treatment of pancreatic cancer $[109,110]$.

Tumor vaccination is another immunotherapeutic strategy on the basis that tumor antigens, both mutated proteins present exclusively on cancer cells and normal proteins present at a higher concentration on cancer cells, are expressed by a remarkable proportion of pancreatic tumors [100]. The vaccines could be administered as whole cells, proteins, peptides, DNA, and RNA, which can cause antigen-specific $\mathrm{T}$ cell responses that may lead to tumor regression. KRAS is one of the most frequent oncogenic mutations present in pancreatic cancers, mostly as a single point mutation at codon 12 resulting in a constitutive activation of KRAS, which can be recognized both by $\mathrm{T}$ helper cells and cytotoxic T-cells and can be used as an antigen in peptide vaccination for tumors with KRAS mutations [111]. In a phase $1 / 2$ clinical trial, a mutant KRAS vaccine, designed to induce T-helper responses, seemed to induce immunologic response in a number of patients with resected pancreatic cancer, and it might be associated with improved long-term immune responses and long-term survival [111]. However, in another study, KRAS vaccination for patients with resected pancreatic cancer failed to show elicitable immunogenicity or proven efficacy, although it is safe and tolerable [112]. Whole tumor cell vaccines have the advantage of containing all possible tumor antigens and can be patient-specific. GVAX pancreas is an allogeneic whole-cell pancreatic cancer vaccine generated from pancreatic cancer cell lines modified to express granulocyte-macrophage colony-stimulating factor (GM-CSF) [113]. The rationale for designing the allogeneic GVAX vaccine is that many tumor antigens are commonly expressed among different patients' cancers, and GM-CSF released by the modified tumor cells induce antigen-presenting cells chemotaxis to the vaccinating tumor, which then phagocytose the tumor cells and present the tumor antigens on MHC class I and II molecules to both CD4+ and CD8+ T cells and thus induce immune-response against the tumor. In several clinical trials, the GVAX pancreas vaccine, in combination with the use of conventional chemotherapy, is shown well-tolerated and induced systemic antitumor immunity to autologous tumor cells in a dose-dependent manner in both resected and advanced pancreatic cancer patients [113-115]. Besides, the use of GVAX is seemingly associated with improved survival and correlated with mesothelin-specific CD8+ T cell responses $[114,115]$. To enhance the efficacy of GVAX pancreas tumor vaccine, cyclophosphamide could be administered to inhibit regulatory T cells [115]. Apart from being used in combination with chemotherapeutic agents, GVAX pancreas could also be administered in combination with CRS-207, a live-attenuated Listeria monocytogenes vaccine expressing mesothelin, which could increase the incidence of mesothelin-specific T cell responses [116]. GVAX/cyclophosphamide in combination with CRS-207 for second-line treatment was suggested to extend survival in patients with metastatic pancreatic cancer [117]. In addition, the combination of GVAX and immune checkpoint inhibitors might provide further benefit to patients with pancreatic cancer and could be evaluated in clinical trials [118,119]. Nevertheless, the use of a telomerase peptide vaccine, GV-1001, in combination with modern chemotherapeutic agents gemcitabine and capecitabine did not show any survival advantage when compared to chemotherapy alone in patients with locally advanced or metastatic pancreatic cancers [120].

Other immunotherapeutic strategies have also been tested in patients with pancreatic cancers; however, most of them failed to show any clinically meaningful effects as had been found in other 
malignancies. Therefore, new strategies of immunotherapy to induce potent immune response to the tumors need to be developed. Investigation in molecular mechanisms that underlie the tumorigenesis of pancreatic cancer may provide novel therapeutic opportunities in these patients and instruct patient selection for optimal treatment.

\subsection{Molecular Pathology of Pancreatic Ductal Adenocarcinoma}

Cancer is a genetic disease caused by the accumulation of somatic mutations in oncogenes and tumor suppressor genes. Genetic analyses reveal four major oncogenes (KRAS, CDKN2A, TP53, $S M A D 4)$, amongst other mutated genes present at lower prevalence, involved in the development of pancreatic ductal adenocarcinoma [121]. Somatic activating mutations of the KRAS oncogene, which encodes a $\sim 21 \mathrm{kDa}$ small GTPase, are present in over $90 \%$ of PDAC patients [122]. In PDACs, the activating mutations of KRAS are mostly point mutations at codon 12, leading to constitutive activation of KRAS protein and persistent stimulation of downstream pathways. Three major KRAS downstream pathways are identified in PDAC, including Raf/Mek/Erk, PI3K/Pdk1/Akt, and the Ral guanine nucleotide exchange factor pathway. In vivo analyses with mouse models demonstrate that sustained oncogenic KRAS signaling is essential for both the progression and maintenance of PDAC and the growth and maintenance of its metastatic lesions [123-125]. Apart from advanced PDAC, somatic KRAS activating mutation is also present in most low-grade pancreatic intraepithelial neoplasms (PanINs) - the most common precursor lesion of PDAC, indicating that KRAS mutation is one of the earliest alterations in the initiation of pancreatic tumorigenesis [126]. However, low frequency of spontaneous progression of precursor lesions to invasive PDAC suggests that additional genetic aberrations (CDKN2A, TP53, or SMAD4) are needed for disease progression [127].

Three major tumor suppressor genes (CDKN2A, TP53, and SMAD4) have been identified frequently mutated in PDAC, and they are strongly associated with malignant behavior of the tumor and may predict poor survival in patients with resectable pancreatic cancers [128]. The cyclin dependent kinase inhibitor 2A (CDKN2A), which functions in cell cycle control by a combination of 2 CDK kinase inhibitor isoforms and 1 alternate open reading frame isoform as p53 protein stabilizer, is the most frequent altered tumor suppressor gene, with loss-of function mutations in more than $90 \%$ of PDAC $[129,130]$. Alterations in CDKN2A are also early events, with loss of protein function in a subset of low-grade PanINs [126,131]. On the contrary, alterations of TP53 and SMAD4 expression are late events, occurring only in high grade PanINs or invasive carcinomas [131,132]. Somatic mutations in the tumor suppressor gene TP53, which responds to diverse cellular stresses by inducing cell cycle arrest and apoptosis, are also frequently shown in PDACs [133]. In addition to pancreatic cancers, mutations in this gene are also associated with a wide range of human cancers. Somatic inactivation of tumor suppressor gene SMAD family member 4 (SMAD4) occurs in greater than 50\% of PDACs [134]. $S M A D 4$ encodes a signal transduction protein downstream of the transforming growth factor $\beta$ (TGF- $\beta$ ) pathway. In response to the activating signaling from TGF- $\beta$, Smad $2 / 3$, the TGF- $\beta$ receptor substrates, are phosphorylated and associated with Smad 4, forming a Smad complex, which then translocates into the nucleus and regulates gene expression by interacting with DNA and DNA-binding proteins [134]. In normal pancreas cells, the TGF- $\beta / S M A D 4$ signaling pathway induces a tumor suppressive effect by activation of cell cycle arrest, apoptosis of epithelial cells, and the maintenance of genomic integrity; whereas, in PDACs, due to inactivation or loss of SMAD4, SMAD4-independent TGF- $\beta$ signaling pathways are triggered, resulting in reduced cell cycle arrest and apoptosis, promotion of epithelial-to-mesenchymal transition and angiogenesis and induction of immune suppression, all of which lead to progression and metastasis of cancer cells [134]. However, studies using genetically engineered mice show that pancreas-specific SMAD4 deficiency does not initiate either PanIN or invasive PDAC; SMAD4 loss markedly promotes tumor progression initiated by KRASG12D activating mutation, indicating that blockade of TGF- $\beta$ signaling and activation of Ras signaling cooperate to promote PDAC progression $[135,136]$. In addition, overexpression of TGF- $\beta$ activates Ras/Erk, P13K/AKt, p38 MAPK, and NF-KB pathways, all of which play a role in PDAC tumorigenesis. 
Apart from these well-characterized oncogene and tumor suppressor genes, other genes are altered at lower prevalence in PDACs but may still play a pivotal role in pancreatic oncogenesis. For instance, GPRC5A, a member of the G protein-coupled receptor family, is shown upregulated in pancreatic cancer primary and metastatic lesions as compared with normal tissues, and it promotes the growth and migration of pancreatic cancer cells [137]. The standard chemotherapy treatment with gemcitabine increased the expression of GPRC $5 A$ by the interaction between its mRNA and RNA-binding protein HuR; whereas, knockout of GPRC5A sensitized pancreatic cancers to gemcitabine chemotherapy. Knockout of GPRC5A reduced the proliferation and migration ability of PDAC cells and suppressed chemotherapy resistance with various chemotherapy agents currently in clinical use, suggesting that targeting GPRC5A may have the potential to improve chemotherapy efficacy [138]. The RIP $1 / 3$ proteins, the main components of the necrosome, are also shown highly expressed in PDAC and are pivotal in the process of necroptosis, another type of programed cell death [139]. The RIP 1/3 promote PDAC oncogenesis and induce the immunosuppressive tumor microenvironment through both the necroptosis-induced CXCL1 and Mincle signaling; whereas, depletion of RIP 1/3, CXCL1, or Mincle protected against PDAC progression and restored anti-tumor immunity in vivo.

Moreover BRCA1 and BRCA2 mutations resulting in DNA damage repair deficiency and increasing especially the risk for breast and ovarian cancer are the most common causes of familial pancreatic cancer. In familial pancreatic cancer, BRCA2 is mutated in about $5 \%$ to $10 \%$ of cases and BRCA1 in approximately $1 \%[140]$.

3.2.1. Molecular Subtypes of Pancreatic Cancers and Other Considerations Based on Genomic and Transcriptomic Analyses

Whole-exome and genome sequencing has revealed that human PDAC is an extremely heterogeneous disease with diverse molecular subtypes [141-147]. Although the major oncogenic mutations are pivotal in the tumorigenesis of PDAC, most other genes are mutated in only a small proportion of tumors. These genes, however, are typically a part of or affect several common signaling pathways. Studies with these cutting-edge analyses show that there are several principal signaling pathways that are genetically altered in most PDACs, but specific genes altered in any individual tumor are largely different $[141,142]$. This molecular heterogeneity of PDACs may explain, at least in part, the reason why agents targeting specific oncogenic genes have mostly failed to benefit unselected patient populations. Thus, drugs targeting a signaling pathway or a key point of the pathway could potentially improve treatment outcomes and warrant further development.

Many studies set out to define molecular subtypes of PDACs based on the genetic mutational characteristics of the tumor to provide evidence for patient selection for optimal treatment [144-147]. In the most recent study conducted by an international research group, Bailey and colleagues, with the use of transcriptomic sequencing, propose 4 molecular subtypes of PDACs: squamous, pancreatic progenitor, aberrantly differentiated endocrine exocrine (ADEX), and immunogenic based on the differential expression of transcription factors and downstream targets [147]. Squamous tumors are characterized by the alteration of gene networks involved in inflammation, hypoxia response, metabolic reprogramming, TGF- $\beta$ signaling, and autophagy. They are also associated with mutations in TP53 and KDM6A, activation of MYC signaling, $\alpha 6 \beta 1$ and $\alpha 6 \beta 4$ integrin signaling, and EGF signaling pathways, upregulation of TP $63 \triangle \mathrm{N}$ network, as well as hypermethylation of pancreatic endodermal cell-fate determining genes (PDX1, MNX1, GATA6, and HNF1B). In addition, pancreatic squamous tumors have a poor prognosis. Pancreatic progenitor tumors are defined by the preferential expression of genes involved in pancreatic endoderm cell-fate determination towards a pancreatic lineage that are pivotal for early pancreatic development, including transcription factors PDX1, MNX1, HNF4G, HNF4A, HNF1B, HNF1A, FOXA2, FOXA3, and HES1. Besides, alterations in genes regulating fatty acid oxidation, steroid hormone biosynthesis, drug metabolism and O-linked glycosylation of mucins, as well as TGFBR2 inactivation are also typical in this subtype. The ADEX class, which is a subclass of the pancreatic progenitor tumors, displays upregulation of transcriptional networks 
involved in later stages of pancreatic development and differentiation. Transcriptional networks involved in both exocrine (NR5A2, MIST1, RBPJL and their downstream targets) and endocrine (INS, NEUROD1, NKX2-2, and MAFA) differentiation at later stages are upregulated, rather than one or the other as is the case in normal pancreas development. The immunogenic subtype, apart from sharing many molecular characteristics with pancreatic progenitor tumors, is associated with upregulated immune networks including B cell signaling pathways, antigen presentation, CD4+ T cell, CD8+ T cell and Toll-like receptor signaling pathways. Additionally, the study also identified 32 recurrently mutated genes aggregating into 10 signaling pathways, including KRAS, TGF- $\beta$, WNT, NOTCH, ROBO/SLIT signalling, G1/S transition, SWI-SNF, chromatin modification, DNA repair and RNA processing. The identification of signaling pathways important in PDAC tumorigenesis and molecular classification provides the most pivotal insights in improving the clinical outcomes of PDAC patients by selecting patients for the optimal therapy.

Recent studies based on novel genomic analysis and informatics methods also challenge the conventional notion of pancreatic cancer progression model [148]. PDAC tumorigenesis may neither be gradual nor follow the currently prevailing model of gene alteration sequence (KRAS, followed by CDKN2A, then TP53 and SMAD4). On the contrary, simultaneous gene rearrangements associated with mitotic errors occur at early stages of tumorigenesis and confer its invasive and metastatic properties, which is supported by the observation that early stage PanIN2 lesions share a great proportion of somatic mutations required for PDAC development with the invasive cancer [149].

\subsubsection{Epigenetic Modifications in the Tumorigenesis of PDACs}

Another concern in the initiation and progression of PDAC is the epigenetic modifications of oncogenes and tumor suppressor genes, which alter the conformation of the chromatin and histones in a reversible manner, leading to changes in gene promoter accessibility and gene expression [150]. The best characterized mechanisms of epigenetic regulations that play a role in the tumorigenesis of PDACs include DNA methylation and histone modifications (such as methylation and acetylation). DNA methylation of tumor suppressor genes $A P C, B R C A 1, C D K N 2 A$ at their promoter regions blocks transcription activity, which is thought to be associated with human PDACs [150]. Genome-wide DNA methylation analysis in PDACs shows that aberrant hypermethylation is prevalent at $5^{\prime}$ regions with reduced mRNA expression levels and is involved in key molecular mechanisms important to PDAC, including TGF- $\beta$, Wnt, integrin signaling, cell adhesion, stellate cell activation, and axon guidance signaling pathways [151]. DNA methylation is carried out by 3 active DNA methyltransferases (DNMTs), including DNMT1, DNMT3A, and DNMT3B, which transfer a methyl group from S-adenosylmethionine to cytosine residues in DNA. All 3 DNMTs have been shown increased in PDACs and are associated with lower overall survival [152,153]. Several DNMT inhibitors have been developed and shown some promising results, but their efficacy in the treatment of pancreatic cancer still needs further investigations [150].

Histone methylation and acetylation are the 2 most important types of histone modifications associated with pancreatic tumorigenesis. Histone methylation is regulated by histone methyltransferases (HMTs) and histone demethylases (HDMs) in a reversible manner, mostly on the lysine residues of histone amino acid side chains [150]. The effect of histone methylation varies depending on the position of methylated lysine residue and the extent of methylation (mono-, di, or tri-methylation). A recent whole genome sequencing and copy number analysis identified KDM6A and MLL2 as the most frequently altered histone methylation regulatory genes in pancreatic cancers [146]. KDM6A is an H3K27me3 demethylase, which is also crucial for pancreas endodermal differentiation through regulating Wnt signaling pathway [154]. Enrichment of H3K27me3 marks on gene promoter regions is associated with suppressed expression of the genes. H3K27 methylation is carried out by the histone methyltransferase EZH2, a component of the polycomb group protein complex, which is upregulated within the cell nucleus in PDAC and possibly contributes to its progression [155]. However, a study with pancreatic tissue sample indicates that H3K27me3 expression is significantly reduced in 
pancreatic cancer as compared to normal tissues and is associated with worse overall survival [156]. On the other hand, MLL2 is a methyltransferase for H3K4 (an activating epigenetic mark) and is shown altered in a smaller proportion of PDACs [156]. Histone acetylation is the most well-studied histone modification. The acetylation of histones, with an acetyl group attached to the lysine residues of histone N-terminal tails, generally results in relaxed chromatin conformation and transcription activation [150]. It is a balanced dynamic process controlled by the reverse regulatory functions of histone acetyltransferases (HATs) and histone deacetylases (HDACs); whereas, aberrant balance of HATs and HDACs is implicated in a variety of human diseases, including pancreatic cancers. HDACs $1,2,3$, and 7 have been found overexpressed in a subset of PDACs, which all play an important role in the development of the disease [157]. However, the functions of HATs have been far less investigated. The HAT p300 seems to possess multifaceted functions in the tumorigenesis of PDACs: it shows decreased expression in highly metastatic PDAC cell lines, suggesting a metastasis-suppressive role in PDACs; and it is also involved in the activation of c-myc gene, suggesting its growth-promoting function in PDACs [157]. Various agents targeting HATs and HDACs have been intensively tested in preclinical and clinical studies, but their effects are generally disappointing [158]. Hence, novel agents with highly selective targeting functions and the underlying molecular mechanisms of epigenetic modifications clearly warrant further exploration.

Additionally, posttranscriptional RNA modification is another aspect of epigenetic regulation. N6-methyladenosine (m6A) is the most abundant mRNA modification in mammals, which is a reversible process regulated by a group of RNA methyltransferases and demethylases, regulating RNA stabilities, mRNA splicing, microRNA processing, and mRNA translation. A recent preclinical study shows that methyltransferase-like 3 (METTL3), an RNA m6A methyltransferase, is involved in the chemo- and radioresistance in pancreatic cancer cells, although it does not show any effect on the morphology and proliferation of the cells [159]. On the other hand, another study shows that ALKBH5, an RNA demethylase, is down-regulated in pancreatic cancer cells, and is associated with the significant enrichment of total RNA methylation in PDAC cells [160]. The cellular and molecular processes of RNA methylation underlying PDAC development still needs further investigation.

Two further proteins playing an important role in epigenetic regulation of PDAC are UNR (Upstream of N-ras) and PIWI (P-element-induced wimpy testis). Low UNR expression was significantly associated with shorter progression-free survival after surgery [161]. Human P-element-induced wimpy testis 1 and 2 (PIWIL1 and PIWIL2) proteins act as protectors of germline and correlate with factors associated to the progenitor molecular subtype of PDAC [162]. Both proteins could be a potential prognostic biomarker for resectable PDAC, improving subsequent adjuvant treatment decisions.

Author Contributions: Conceptualization, M.B., Z.W., and G.F.W.; writing-original draft preparation, M.B., W.Z., and G.F.W.; writing — review and editing, C.K., C.P., R.G., and G.F.W. All authors read and approved the final manuscript.

Funding: This research received no external funding.

Conflicts of Interest: The authors declare no conflict of interest.

\author{
Abbreviations \\ PDAC Pancreatic ductal adenocarcinoma \\ EUS-FNA Endoscopic ultrasound-guided fine needle aspiration \\ CRT Chemoradiotherapy \\ ECOG Eastern Co-operative Oncology Group \\ NK cells Natural killer cells \\ APC Antigen presenting cells \\ MHC Major histocompatibility complex \\ PD-1 Programed cell death-1 \\ CTLA-4 Cytotoxic T-lymphocyte associated protein-4 \\ FAP Fibroblast-associated protein
}




$\begin{array}{ll}\text { CAF } & \text { Cancer-associated fibroblast } \\ \text { GM-CSF } & \text { Granulocyte-macrophage colony-stimulating factor } \\ \text { PanIN } & \text { Pancreatic intraepithelial neoplasm } \\ \text { CDKN2A } & \text { Cyclin dependent kinase inhibitor 2A } \\ \text { TGF- } \beta & \text { Transforming growth factor } \beta \\ \text { ADEX } & \text { Aberrantly differentiated endocrine exocrine } \\ \text { HMT } & \text { Histone methyltransferase } \\ \text { HDM } & \text { Histone demethylase } \\ \text { HAT } & \text { Histone acetyltransferase } \\ \text { HDAC } & \text { Histone deacetylase } \\ \text { UNR } & \text { Upstream of N-ras } \\ \text { PIWI } & \text { P-element-induced wimpy testis }\end{array}$

\section{References}

1. Bray, F.; Ferlay, J.; Soerjomataram, I.; Siegel, R.L.; Torre, L.A.; Jemal, A. Global cancer statistics 2018: GLOBOCAN estimates of incidence and mortality worldwide for 36 cancers in 185 countries. CA A Cancer J. Clin. 2018, 68, 394-424. [CrossRef] [PubMed]

2. Siegel, R.L.; Miller, K.D.; Jemal, A. Cancer statistics, 2019. CA A Cancer J. Clin. 2019, 69, 7-34. [CrossRef] [PubMed]

3. Kamisawa, T.; Wood, L.D.; Itoi, T.; Takaori, K. Pancreatic cancer. Lancet 2016, 388, 73-85. [CrossRef]

4. Keane, M.G.; Horsfall, L.; Rait, G.; Pereira, S.P. A case-Control study comparing the incidence of early symptoms in pancreatic and biliary tract cancer. BMJ Open 2014, 4, 005720. [CrossRef] [PubMed]

5. Rhim, A.D.; Mirek, E.T.; Aiello, N.M.; Maitra, A.; Bailey, J.M.; McCallister, F.; Reichert, M.; Beatty, G.L.; Rustgi, A.K.; Vonderheide, R.H.; et al. EMT and dissemination precede pancreatic tumor formation. Cell 2012, 148, 349-361. [CrossRef] [PubMed]

6. Giuliano, K.; Ejaz, A.; He, J. Technical aspects of pancreaticoduodenectomy and their outcomes. Chin. Clin. Oncol. 2017, 6, 901. [CrossRef] [PubMed]

7. Conlon, K.C.; Klimstra, D.S.; Brennan, M.F. Long-term survival after curative resection for pancreatic ductal adenocarcinoma. Clinicopathologic analysis of 5-year survivors. Ann. Surg. 1996, 223, 273-279. [CrossRef] [PubMed]

8. Howard, T.; Krug, J.; Yu, J.; Zyromski, N.; Schmidt, C.; Jacobson, L.; Madura, J.; Wiebke, E.; Lillemoe, K. A Margin-Negative R0 Resection Accomplished with Minimal Postoperative Complications Is the Surgeon's Contribution to Long-Term Survival in Pancreatic Cancer. J. Gastrointest. Surg. 2006, 10, 1338-1346. [CrossRef] [PubMed]

9. Quarto, G.; Perrotta, S.; Furino, E.; Amato, B.; Bianco, T.; De Palma, G.; Forestieri, P.; Benassai, G.; Benassai, G.L.; Benassai, G. Long-term survival after curative resection for pancreatic ductal adenocarcinoma-Surgical treatment. Int. J. Surg. 2015, 21, S1-S3.

10. La Torre, M.; Nigri, G.; Ferrari, L.; Cosenza, G.; Ravaioli, M.; Ramacciato, G. Hospital volume, margin status, and long-term survival after pancreaticoduodenectomy for pancreatic adenocarcinoma. Am. Surg. 2012, 78, 225-229.

11. Nimptsch, U.; Krautz, C.; Weber, G.F.; Mansky, T.; Grützmann, R. Nationwide In-hospital Mortality Following Pancreatic Surgery in Germany is Higher than Anticipated. Ann. Surg. 2016, 264, 1-1090. [CrossRef] [PubMed]

12. Krautz, C.; Nimptsch, U.; Weber, G.F.; Mansky, T.; Grützmann, R. Effect of Hospital Volume on In-hospital Morbidity and Mortality Following Pancreatic Surgery in Germany. Ann. Surg. 2018, 267, 411-417. [CrossRef] [PubMed]

13. Bakkevold, K.; Arnesjø, B.; Dahl, O.; Kambestad, B. Adjuvant combination chemotherapy (AMF) following radical resection of carcinoma of the pancreas and papilla of vater-Results of a controlled, prospective, randomised multicentre study. Eur. J. Cancer 1993, 29, 698-703. [CrossRef]

14. Study Group of Surgical Adjuvant Therapy for Carcinomas of the Pancreas and Biliary Tract; Takada, T.; Amano, H.; Yasuda, H.; Nimura, Y.; Matsushiro, T.; Kato, H.; Nagakawa, T.; Nakayama, T. Is postoperative adjuvant chemotherapy useful for gallbladder carcinoma? A phase III multicenter prospective randomized controlled trial in patients with resected pancreaticobiliary carcinoma. Cancer 2002, 95, 1685-1695. [PubMed] 
15. Neoptolemos, J.P.; Stocken, D.D.; Friess, H.; Bassi, C.; Dunn, J.A.; Hickey, H.; Beger, H.; Fernandez-Cruz, L.; Dervenis, C.; Lacaine, F.; et al. A Randomized Trial of Chemoradiotherapy and Chemotherapy after Resection of Pancreatic Cancer. N. Engl. J. Med. 2004, 350, 1200-1210. [CrossRef]

16. Kosuge, T.; Kiuchi, T.; Mukai, K.; Kakizoe, T. A Multicenter Randomized Controlled Trial to Evaluate the Effect of Adjuvant Cisplatin and 5-Fluorouracil Therapy after Curative Resection in Cases of Pancreatic Cancer. Jpn. J. Clin. Oncol. 2006, 36, 159-165. [CrossRef]

17. Oettle, H.; Neuhaus, P.; Hochhaus, A.; Hartmann, J.T.; Gellert, K.; Ridwelski, K.; Niedergethmann, M.; Zülke, C.; Fahlke, J.; Arning, M.B.; et al. Adjuvant chemotherapy with gemcitabine and long-term outcomes among patients with resected pancreatic cancer: The CONKO-001 randomized trial. JAMA 2013, 310, 1473-1481. [CrossRef]

18. Oettle, H.; Post, S.; Neuhaus, P.; Gellert, K.; Langrehr, J.; Ridwelski, K.; Schramm, H.; Fahlke, J.; Zuelke, C.; Burkart, C.; et al. Adjuvant chemotherapy with gemcitabine vs. observation in patients undergoing curative-intent resection of pancreatic cancer: A randomized controlled trial. JAMA 2007, 297, 267-277. [CrossRef]

19. Yoshitomi, H.; Togawa, A.; Kimura, F.; Ito, H.; Shimizu, H.; Yoshidome, H.; Otsuka, M.; Kato, A.; Nozawa, S.; Furukawa, K.; et al. A randomized phase II trial of adjuvant chemotherapy with uracil/tegafur and gemcitabine versus gemcitabine alone in patients with resected pancreatic cancer. Cancer 2008, 113, 2448-2456. [CrossRef]

20. Neoptolemos, J.P.; Stocken, D.D.; Smith, C.T.; Bassi, C.; Ghaneh, P.; Owen, E.; Moore, M.; Padbury, R.; Doi, R.; Smith, D.; et al. Adjuvant 5-fluorouracil and folinic acid vs observation for pancreatic cancer: Composite data from the ESPAC-1 and -3(v1) trials. Br. J. Cancer 2009, 100, 246-250. [CrossRef]

21. Ueno, H.; Kosuge, T.; Matsuyama, Y.; Yamamoto, J.; Nakao, A.; Egawa, S.; Doi, R.; Monden, M.; Hatori, T.; Tanaka, M.; et al. A randomised phase III trial comparing gemcitabine with surgery-only in patients with resected pancreatic cancer: Japanese Study Group of Adjuvant Therapy for Pancreatic Cancer. Br. J. Cancer 2009, 101, 908-915. [CrossRef] [PubMed]

22. Neoptolemos, J.P.; Stocken, D.D.; Bassi, C.; Ghaneh, P.; Cunningham, D.; Goldstein, D.; Padbury, R.; Moore, M.J.; Gallinger, S.; Mariette, C.; et al. Adjuvant chemotherapy with fluorouracil plus folinic acid vs. gemcitabine following pancreatic cancer resection: A randomized controlled trial. JAMA 2010, 304, 1073-1081. [CrossRef] [PubMed]

23. Regine, W.F.; Winter, K.A.; Abrams, R.; Safran, H.; Hoffman, J.P.; Konski, A.; Benson, A.B.; Macdonald, J.S.; Rich, T.A.; Willett, C.G. Fluorouracil-based chemoradiation with either gemcitabine or fluorouracil chemotherapy after resection of pancreatic adenocarcinoma: 5-year analysis of the U.S. Intergroup/RTOG 9704 phase III trial. Ann. Surg. Oncol. 2011, 18, 1319-1326. [CrossRef] [PubMed]

24. Regine, W.F.; Winter, K.A.; Abrams, R.A.; Safran, H.; Hoffman, J.P.; Konski, A.; Benson, A.B.; Macdonald, J.S.; Kudrimoti, M.R.; Fromm, M.L.; et al. Fluorouracil vs. gemcitabine chemotherapy before and after fluorouracil-based chemoradiation following resection of pancreatic adenocarcinoma: A randomized controlled trial. JAMA 2008, 299, 1019-1026. [CrossRef] [PubMed]

25. Reni, M.; Balzano, G.; Aprile, G.; Cereda, S.; Passoni, P.; Zerbi, A.; Tronconi, M.C.; Milandri, C.; Saletti, P.; Rognone, A.; et al. Adjuvant PEFG (Cisplatin, Epirubicin, 5-Fluorouracil, Gemcitabine) or Gemcitabine Followed by Chemoradiation in Pancreatic Cancer: A Randomized Phase II Trial. Ann. Surg. Oncol. 2012, 19, 2256-2263. [CrossRef] [PubMed]

26. Shimoda, M.; Kubota, K.; Shimizu, T.; Katoh, M. Randomized clinical trial of adjuvant chemotherapy with S-1 versus gemcitabine after pancreatic cancer resection. Br. J. Surg. 2015, 102, 746-754. [CrossRef] [PubMed]

27. Uesaka, K.; Boku, N.; Fukutomi, A.; Okamura, Y.; Konishi, M.; Matsumoto, I.; Kaneoka, Y.; Shimizu, Y.; Nakamori, S.; Sakamoto, H.; et al. Adjuvant chemotherapy of S-1 versus gemcitabine for resected pancreatic cancer: A phase 3, open-label, randomised, non-inferiority trial (JASPAC 01). Lancet 2016, 388, 248-257. [CrossRef]

28. Neoptolemos, J.P.; Palmer, D.H.; Ghaneh, P.; Psarelli, E.E.; Valle, J.W.; Halloran, C.M.; Faluyi, O.; O’Reilly, D.A.; Cunningham, D.; Wadsley, J.; et al. Comparison of adjuvant gemcitabine and capecitabine with gemcitabine monotherapy in patients with resected pancreatic cancer (ESPAC-4): A multicentre, open-label, randomised, phase 3 trial. Lancet 2017, 389, 1011-1024. [CrossRef] 
29. Sinn, M.; Bahra, M.; Liersch, T.; Gellert, K.; Messmann, H.; Bechstein, W.; Waldschmidt, D.; Jacobasch, L.; Wilhelm, M.; Rau, B.M.; et al. CONKO-005: Adjuvant Chemotherapy with Gemcitabine Plus Erlotinib Versus Gemcitabine Alone in Patients After R0 Resection of Pancreatic Cancer: A Multicenter Randomized Phase III Trial. J. Clin. Oncol. 2017, 35, 3330-3337. [CrossRef]

30. Reni, M.; Balzano, G.; Zanon, S.; Zerbi, A.; Rimassa, L.; Castoldi, R.; Pinelli, D.; Mosconi, S.; Doglioni, C.; Chiaravalli, M.; et al. Safety and efficacy of preoperative or postoperative chemotherapy for resectable pancreatic adenocarcinoma (PACT-15): A randomised, open-label, phase 2-3 trial. Lancet Gastroenterol. Hepatol. 2018, 3, 413-423. [CrossRef]

31. Kooby, D.A.; Gillespie, T.W.; Liu, Y.; Byrd-Sellers, J.; Landry, J.; Bian, J.; Lipscomb, J. Impact of Adjuvant Radiotherapy on Survival after Pancreatic Cancer Resection: An Appraisal of Data from the National Cancer Data Base. Ann. Surg. Oncol. 2013, 20, 3634-3642. [CrossRef] [PubMed]

32. Morganti, A.G.; Falconi, M.; Van Stiphout, R.G.; Mattiucci, G.-C.; Alfieri, S.; Calvo, F.A.; Dubois, J.-B.; Fastner, G.; Herman, J.M.; Maidment, B.W.; et al. Multi-institutional Pooled Analysis on Adjuvant Chemoradiation in Pancreatic Cancer. Int. J. Radiat. Oncol. 2014, 90, 911-917. [CrossRef] [PubMed]

33. Rutter, C.E.; Park, H.S.; Corso, C.D.; Lester-Coll, N.H.; Mancini, B.R.; Yeboa, D.N.; Johung, K.L. Addition of radiotherapy to adjuvant chemotherapy is associated with improved overall survival in resected pancreatic adenocarcinoma: An analysis of the National Cancer Data Base. Cancer 2015, 121, 4141-4149. [CrossRef] [PubMed]

34. Sugawara, A.; Kunieda, E. Effect of adjuvant radiotherapy on survival in resected pancreatic cancer: A propensity score surveillance, epidemiology, and end results database analysis. J. Surg. Oncol. 2014, 110, 960-966. [CrossRef] [PubMed]

35. Liao, W.-C.; Chien, K.-L.; Lin, Y.-L.; Wu, M.-S.; Lin, J.-T.; Wang, H.-P.; Tu, Y.-K. Adjuvant treatments for resected pancreatic adenocarcinoma: A systematic review and network meta-analysis. Lancet Oncol. 2013, 14, 1095-1103. [CrossRef]

36. Palmer, D.H.; Stocken, D.D.; Hewitt, H.; Markham, C.E.; Hassan, A.B.; Johnson, P.J.; Buckels, J.A.C.; Bramhall, S.R. A Randomized Phase 2 Trial of Neoadjuvant Chemotherapy in Resectable Pancreatic Cancer: Gemcitabine Alone Versus Gemcitabine Combined with Cisplatin. Ann. Surg. Oncol. 2007, 14, 2088-2096. [CrossRef] [PubMed]

37. Sahora, K.; Schindl, M.; Kuehrer, I.; Eisenhut, A.; Werba, G.; Brostjan, C.; Telek, B.; Ba'Ssalamah, A.; Stift, J.; Schoppmann, S.F.; et al. A phase II trial of two durations of Bevacizumab added to neoadjuvant gemcitabine for borderline and locally advanced pancreatic cancer. Anticancer Res. 2014, 34, 2377-2384.

38. Landry, J.; Catalano, P.J.; Staley, C.; Harris, W.; Hoffman, J.; Talamonti, M.; Xu, N.; Cooper, H.; Benson, A.B., III. Randomized phase II study of gemcitabine plus radiotherapy versus gemcitabine, 5-fluorouracil, and cisplatin followed by radiotherapy and 5-fluorouracil for patients with locally advanced, potentially resectable pancreatic adenocarcinoma. J. Surg. Oncol. 2010, 101, 587-592. [CrossRef] [PubMed]

39. Golcher, H.; Brunner, T.B.; Witzigmann, H.; Marti, L.; Bechstein, W.O.; Bruns, C.; Jungnickel, H.; Schreiber, S.; Grabenbauer, G.G.; Meyer, T.; et al. Neoadjuvant chemoradiation therapy with gemcitabine/cisplatin and surgery versus immediate surgery in resectable pancreatic cancer. Strahlenther. Onkol. 2015, 191, 7-16. [CrossRef]

40. Casadei, R.; Di Marco, M.; Ricci, C.; Santini, D.; Serra, C.; Calculli, L.; D’Ambra, M.; Guido, A.; Morselli-Labate, A.M.; Minni, F. Neoadjuvant Chemoradiotherapy and Surgery Versus Surgery Alone in Resectable Pancreatic Cancer: A Single-Center Prospective, Randomized, Controlled Trial Which Failed to Achieve Accrual Targets. J. Gastrointest. Surg. 2015, 19, 1802-1812. [CrossRef]

41. Tzeng, C.-W.D.; Fleming, J.B.; Lee, J.E.; Wang, X.; Pisters, P.W.T.; Vauthey, J.-N.; Varadhachary, G.; Wolff, R.A.; Katz, M.H.G. Yield of clinical and radiographic surveillance in patients with resected pancreatic adenocarcinoma following multimodal therapy. HPB 2012, 14, 365-372. [CrossRef] [PubMed]

42. Tzeng, C.-W.D.; Abbott, D.E.; Cantor, S.B.; Fleming, J.B.; Lee, J.E.; Pisters, P.W.T.; Varadhachary, G.R.; Abbruzzese, J.L.; Wolff, R.A.; Ahmad, S.A.; et al. Frequency and Intensity of Postoperative Surveillance After Curative Treatment of Pancreatic Cancer: A Cost-Effectiveness Analysis. Ann. Surg. Oncol. 2013, 20, 2197-2203. [CrossRef] [PubMed] 
43. Schorn, S.; Demir, I.E.; Reyes, C.M.; Saricaoglu, C.; Samm, N.; Schirren, R.; Tieftrunk, E.; Hartmann, D.; Friess, H.; Ceyhan, G.O. The impact of neoadjuvant therapy on the histopathological features of pancreatic ductal adenocarcinoma-A systematic review and meta-analysis. Cancer Treat. Rev. 2017, 55, 96-106. [CrossRef] [PubMed]

44. Lutfi, W.; Talamonti, M.S.; Kantor, O.; Wang, C.-H.; Liederbach, E.; Stocker, S.J.; Bentrem, D.J.; Roggin, K.K.; Winchester, D.J.; Marsh, R.; et al. Perioperative chemotherapy is associated with a survival advantage in early stage adenocarcinoma of the pancreatic head. Surgery 2016, 160, 714-724. [CrossRef] [PubMed]

45. Shubert, C.R.; Bergquist, J.R.; Groeschl, R.T.; Habermann, E.B.; Wilson, P.M.; Truty, M.J.; Smoot, R.L.; Kendrick, M.L.; Nagorney, D.M.; Farnell, M.B. Overall survival is increased among stage III pancreatic adenocarcinoma patients receiving neoadjuvant chemotherapy compared to surgery first and adjuvant chemotherapy: An intention to treat analysis of the National Cancer Database. Surgery 2016, 160, 1080-1096. [CrossRef]

46. Verma, V.; Li, J.; Lin, C. Neoadjuvant Therapy for Pancreatic Cancer: Systematic Review of Postoperative Morbidity, Mortality, and Complications. Am. J. Clin. Oncol. 2016, 39, 302-313. [CrossRef]

47. Burris, H.; Moore, M.J.; Andersen, J.; Green, M.R.; Rothenberg, M.L.; Modiano, M.R.; Christine Cripps, M.; Portenoy, R.K.; Storniolo, A.M.; Tarassoff, P. Improvements in survival and clinical benefit with gemcitabine as first-line therapy for patients with advanced pancreas cancer: A randomized trial. J. Clin. Oncol. 1997, 15, 2403-2413. [CrossRef]

48. Huguier, M.; Barrier, A.; Valinas, R.; Flahault, A.; Adloff, M.; Pezet, D.; Jaeck, D.; Millat, B. Randomized trial of 5-fluorouracil, leucovorin and cisplatin in advanced pancreatic cancer. Hepatogastroenterology 2001, 48, 875-878.

49. Ducreux, M.; Rougier, P.; Pignon, J.-P.; Douillard, J.-Y.; Seitz, J.-F.; Bugat, R.; Bosset, J.-F.; Merouche, Y.; Raoul, J.-L.; Ychou, M.; et al. A randomised trial comparing 5-FU with 5-FU plus cisplatin in advanced pancreatic carcinoma. Ann. Oncol. 2002, 13, 1185-1191. [CrossRef]

50. Colucci, G.; Giuliani, F.; Gebbia, V.; Biglietto, M.; Rabitti, P.; Uomo, G.; Cigolari, S.; Testa, A.; Maiello, E.; Lopez, M. Gemcitabine alone or with cisplatin for the treatment of patients with locally advanced and/or metastatic pancreatic carcinoma: A prospective, randomized phase III study of the Gruppo Oncologia dell'Italia Meridionale. Cancer 2002, 94, 902-910. [CrossRef]

51. Scheithauer, W.; Schüll, B.; Ulrich-Pur, H.; Raderer, M.; Haider, K.; Depisch, D.; Kornek, G.V.; Schmid, K.; Kwasny, W.; Schneeweiss, B.; et al. Biweekly high-dose gemcitabine alone or in combination with capecitabine in patients with metastatic pancreatic adenocarcinoma: A randomized phase II trial. Ann. Oncol. 2003, 14, 97-104. [CrossRef] [PubMed]

52. Tempero, M.; Plunkett, W.; Van Haperen, V.R.; Hainsworth, J.; Hochster, H.; Lenzi, R.; Abbruzzese, J. Randomized Phase II Comparison of Dose-Intense Gemcitabine: Thirty-Minute Infusion and Fixed Dose Rate Infusion in Patients with Pancreatic Adenocarcinoma. J. Clin. Oncol. 2003, 21, 3402-3408. [CrossRef] [PubMed]

53. Ducreux, M.; Mitry, E.; Ould-Kaci, M.; Boige, V.; Seitz, J.F.; Bugat, R.; Breau, J.L.; Bouché, O.; Etienne, P.L.; Tigaud, J.M.; et al. Randomized phase II study evaluating oxaliplatin alone, oxaliplatin combined with infusional 5-FU, and infusional 5-FU alone in advanced pancreatic carcinoma patients. Ann. Oncol. 2004, 15, 467-473. [CrossRef] [PubMed]

54. Louvet, C. Gemcitabine in Combination with Oxaliplatin Compared with Gemcitabine Alone in Locally Advanced or Metastatic Pancreatic Cancer: Results of a GERCOR and GISCAD Phase III Trial. J. Clin. Oncol. 2005, 23, 3509-3516. [CrossRef] [PubMed]

55. Heinemann, V.; Quietzsch, D.; Gieseler, F.; Gonnermann, M.; Schönekäs, H.; Rost, A.; Neuhaus, H.; Haag, C.; Clemens, M.; Heinrich, B.; et al. Randomized Phase III Trial of Gemcitabine Plus Cisplatin Compared with Gemcitabine Alone in Advanced Pancreatic Cancer. J. Clin. Oncol. 2006, 24, 3946-3952. [CrossRef] [PubMed]

56. Moore, M.J.; Goldstein, D.; Hamm, J.; Figer, A.; Hecht, J.R.; Gallinger, S.; Au, H.J.; Murawa, P.; Walde, D.; Wolff, R.A.; et al. Erlotinib Plus Gemcitabine Compared with Gemcitabine Alone in Patients with Advanced Pancreatic Cancer: A Phase III Trial of the National Cancer Institute of Canada Clinical Trials Group. J. Clin. Oncol. 2007, 25, 1960-1966. [CrossRef] [PubMed] 
57. Herrmann, R.; Bodoky, G.; Ruhstaller, T.; Glimelius, B.; Bajetta, E.; Schüller, J.; Saletti, P.; Bauer, J.; Figer, A.; Pestalozzi, B.; et al. Gemcitabine Plus Capecitabine Compared with Gemcitabine Alone in Advanced Pancreatic Cancer: A Randomized, Multicenter, Phase III Trial of the Swiss Group for Clinical Cancer Research and the Central European Cooperative Oncology Group. J. Clin. Oncol. 2007, 25, 2212-2217. [CrossRef] [PubMed]

58. Boeck, S.; Hoehler, T.; Seipelt, G.; Mahlberg, R.; Wein, A.; Hochhaus, A.; Boeck, H.-P.; Schmid, B.; Kettner, E.; Stauch, M.; et al. Capecitabine plus oxaliplatin (CapOx) versus capecitabine plus gemcitabine (CapGem) versus gemcitabine plus oxaliplatin (mGemOx): Final results of a multicenter randomized phase II trial in advanced pancreatic cancer. Ann. Oncol. 2008, 19, 340-347. [CrossRef] [PubMed]

59. Cunningham, D.; Chau, I.; Stocken, D.D.; Valle, J.W.; Smith, D.; Steward, W.; Harper, P.G.; Dunn, J.; Tudur-Smith, C.; West, J.; et al. Phase III Randomized Comparison of Gemcitabine Versus Gemcitabine Plus Capecitabine in Patients with Advanced Pancreatic Cancer. J. Clin. Oncol. 2009, 27, 5513-5518. [CrossRef]

60. Poplin, E.; Feng, Y.; Berlin, J.; Rothenberg, M.L.; Hochster, H.; Mitchell, E.; Alberts, S.; O’Dwyer, P.; Haller, D.; Catalano, P.; et al. Phase III, randomized study of gemcitabine and oxaliplatin versus gemcitabine (fixed-dose rate infusion) compared with gemcitabine (30-min infusion) in patients with pancreatic carcinoma E6201: A trial of the Eastern Cooperative Oncology Group. J. Clin. Oncol. 2009, 27, 3778-3785. [CrossRef] [PubMed]

61. Kulke, M.H.; Tempero, M.A.; Niedzwiecki, D.; Hollis, D.R.; Kindler, H.L.; Cusnir, M.; Enzinger, P.C.; Gorsch, S.M.; Goldberg, R.M.; Mayer, R.J. Randomized Phase II Study of Gemcitabine Administered at a Fixed Dose Rate or in Combination with Cisplatin, Docetaxel, or Irinotecan in Patients with Metastatic Pancreatic Cancer: CALGB 89904. J. Clin. Oncol. 2009, 27, 5506-5512. [CrossRef] [PubMed]

62. Colucci, G.; Labianca, R.; Di Costanzo, F.; Gebbia, V.; Cartenì, G.; Massidda, B.; Dapretto, E.; Manzione, L.; Piazza, E.; Sannicolo, M.; et al. Randomized Phase III Trial of Gemcitabine Plus Cisplatin Compared with Single-Agent Gemcitabine as First-Line Treatment of Patients with Advanced Pancreatic Cancer: The GIP-1 Study. J. Clin. Oncol. 2010, 28, 1645-1651. [CrossRef] [PubMed]

63. Dahan, L.; Bonnetain, F.; Ychou, M.; Mitry, E.; Gasmi, M.; Raoul, J.-L.; Cattan, S.; Phelip, J.-M.; Hammel, P.; Chauffert, B.; et al. Combination 5-fluorouracil, folinic acid and cisplatin (LV5FU2-CDDP) followed by gemcitabine or the reverse sequence in metastatic pancreatic cancer: Final results of a randomised strategic phase III trial (FFCD 0301). Gut 2010, 59, 1527-1534. [CrossRef] [PubMed]

64. Conroy, T.; Desseigne, F.; Ychou, M.; Bouche, O.; Guimbaud, R.; Bécouarn, Y.; Adenis, A.; Raoul, J.-L.; Gourgou-Bourgade, S.; De La Fouchardiere, C.; et al. FOLFIRINOX versus Gemcitabine for Metastatic Pancreatic Cancer. N. Engl. J. Med. 2011, 364, 1817-1825. [CrossRef] [PubMed]

65. Ozaka, M.; Matsumura, Y.; Ishii, H.; Omuro, Y.; Itoi, T.; Mouri, H.; Hanada, K.; Kimura, Y.; Maetani, I.; Okabe, Y.; et al. Randomized phase II study of gemcitabine and S-1 combination versus gemcitabine alone in the treatment of unresectable advanced pancreatic cancer (Japan Clinical Cancer Research Organization PC-01 study). Cancer Chemother. Pharmacol. 2012, 69, 1197-1204. [CrossRef] [PubMed]

66. Nakai, Y.; Isayama, H.; Sasaki, T.; Sasahira, N.; Tsujino, T.; Toda, N.; Kogure, H.; Matsubara, S.; Ito, Y.; Togawa, O.; et al. A multicentre randomised phase II trial of gemcitabine alone vs gemcitabine and S-1 combination therapy in advanced pancreatic cancer: GEMSAP study. Br. J. Cancer 2012, 106, 1934-1939. [CrossRef] [PubMed]

67. Chao, Y.; Wu, C.-Y.; Wang, J.P.; Lee, R.-C.; Lee, W.-P.; Li, C.-P. A randomized controlled trial of gemcitabine plus cisplatin versus gemcitabine alone in the treatment of metastatic pancreatic cancer. Cancer Chemother. Pharmacol. 2013, 72, 637-642. [CrossRef] [PubMed]

68. Von Hoff, D.D.; Ervin, T.; Arena, F.P.; Chiorean, E.G.; Infante, J.; Moore, M.; Seay, T.; Tjulandin, S.A.; Ma, W.W.; Saleh, M.N.; et al. Increased Survival in Pancreatic Cancer with nab-Paclitaxel plus Gemcitabine. N. Engl. J. Med. 2013, 369, 1691-1703. [CrossRef] [PubMed]

69. Ueno, H.; Ioka, T.; Ikeda, M.; Ohkawa, S.; Yanagimoto, H.; Boku, N.; Fukutomi, A.; Sugimori, K.; Baba, H.; Yamao, K.; et al. Randomized Phase III Study of Gemcitabine Plus S-1, S-1 Alone, or Gemcitabine Alone in Patients with Locally Advanced and Metastatic Pancreatic Cancer in Japan and Taiwan: GEST Study. J. Clin. Oncol. 2013, 31, 1640-1648. [CrossRef] [PubMed]

70. Okusaka, T.; Miyakawa, H.; Fujii, H.; Nakamori, S.; Satoh, T.; Hamamoto, Y.; Ito, T.; Maguchi, H.; Matsumoto, S.; Ueno, H.; et al. Updated results from GEST study: A randomized, three-arm phase III study for advanced pancreatic cancer. J. Cancer Res. Clin. Oncol. 2017, 143, 1053-1059. [CrossRef] [PubMed] 
71. Sudo, K.; Ishihara, T.; Hirata, N.; Ozawa, F.; Ohshima, T.; Azemoto, R.; Shimura, K.; Nihei, T.; Nishino, T.; Nakagawa, A.; et al. Randomized controlled study of gemcitabine plus S-1 combination chemotherapy versus gemcitabine for unresectable pancreatic cancer. Cancer Chemother. Pharmacol. 2014, 73, 389-396. [CrossRef] [PubMed]

72. Petrioli, R.; Roviello, G.; Fiaschi, A.I.; Laera, L.; Marrelli, D.; Roviello, F.; Francini, E. Gemcitabine, oxaliplatin, and capecitabine (GEMOXEL) compared with gemcitabine alone in metastatic pancreatic cancer: A randomized phase II study. Cancer Chemother. Pharmacol. 2015, 75, 683-690. [CrossRef] [PubMed]

73. Wang, J.P.; Wu, C.-Y.; Yeh, Y.-C.; Shyr, Y.-M.; Wu, Y.-Y.; Kuo, C.-Y.; Hung, Y.-P.; Chen, M.-H.; Lee, W.-P.; Luo, J.-C.; et al. Erlotinib is effective in pancreatic cancer with epidermal growth factor receptor mutations: A randomized, open-label, prospective trial. Oncotarget 2015, 6, 18162-18173. [CrossRef] [PubMed]

74. Lee, H.S.; Chung, M.J.; Park, J.Y.; Bang, S.; Park, S.W.; Kim, H.G.; Noh, M.H.; Lee, S.H.; Kim, Y.-T.; Kim, H.J.; et al. A randomized, multicenter, phase III study of gemcitabine combined with capecitabine versus gemcitabine alone as first-line chemotherapy for advanced pancreatic cancer in South Korea. Medicine 2017, 96, e5702. [CrossRef] [PubMed]

75. Nagrial, A.; Sjoquist, K.; O'Connor, C.A.; Chantrill, L.; Biankin, A.V.; Scholten, R.J.; Yip, D.; Chin, V. Chemotherapy and radiotherapy for advanced pancreatic cancer. Cochrane Database Syst. Rev. 2018, $2018,3$.

76. Huguet, F.; Girard, N.; Guerche, C.S.-E.; Hennequin, C.; Mornex, F.; Azria, D.; Guerche, C.S.-E. Chemoradiotherapy in the Management of Locally Advanced Pancreatic Carcinoma: A Qualitative Systematic Review. J. Clin. Oncol. 2009, 27, 2269-2277. [CrossRef]

77. Chauffert, B.; Mornex, F.; Bonnetain, F.; Rougier, P.; Mariette, C.; Bouche, O.; Bosset, J.F.; Aparicio, T.; Mineur, L.; Azzedine, A.; et al. Phase III trial comparing intensive induction chemoradiotherapy (60 Gy, infusional 5-FU and intermittent cisplatin) followed by maintenance gemcitabine with gemcitabine alone for locally advanced unresectable pancreatic cancer. Definitive results of the 2000-01 FFCD/SFRO study. Ann. Oncol. 2008, 19, 1592-1599.

78. Loehrer, P.J., Sr.; Feng, Y.; Cardenes, H.; Wagner, L.; Brell, J.M.; Cella, D.; Flynn, P.; Ramanathan, R.K.; Crane, C.H.; Alberts, S.R.; et al. Gemcitabine alone versus gemcitabine plus radiotherapy in patients with locally advanced pancreatic cancer: An Eastern Cooperative Oncology Group trial. J. Clin. Oncol. 2011, 29, 4105-4112. [CrossRef]

79. Hammel, P.; Huguet, F.; van Laethem, J.L.; Goldstein, D.; Glimelius, B.; Artru, P.; Borbath, I.; Bouché, O.; Shannon, J.; André, T.; et al. Effect of Chemoradiotherapy vs. Chemotherapy on Survival in Patients with Locally Advanced Pancreatic Cancer Controlled After 4 Months of Gemcitabine with or Without Erlotinib: The LAP07 Randomized Clinical Trial. JAMA 2016, 315, 1844-1853. [CrossRef]

80. Li, C.-P.; Chao, Y.; Chi, K.-H.; Chan, W.-K.; Teng, H.-C.; Lee, R.-C.; Chang, F.-Y.; Lee, S.-D.; Yen, S.-H. Concurrent chemoradiotherapy treatment of locally advanced pancreatic cancer: Gemcitabine versus 5-fluorouracil, a randomized controlled study. Int. J. Radiat. Oncol. 2003, 57, 98-104. [CrossRef]

81. Wilkowski, R.; Boeck, S.; Ostermaier, S.; Sauer, R.; Herbst, M.; Fietkau, R.; Flentje, M.; Miethe, S.; Boettcher, H.D.; Scholten, T.; et al. Chemoradiotherapy with concurrent gemcitabine and cisplatin with or without sequential chemotherapy with gemcitabine/cisplatin vs chemoradiotherapy with concurrent 5-fluorouracil in patients with locally advanced pancreatic cancer-A multi-centre randomised phase II study. Br. J. Cancer 2009, 101, 1853-1859. [PubMed]

82. Mukherjee, S.; Hurt, C.N.; Bridgewater, J.; Falk, S.; Cummins, S.; Wasan, H.; Crosby, T.; Jephcott, C.; Roy, R.; Radhakrishna, G.; et al. Gemcitabine-based or capecitabine-based chemoradiotherapy for locally advanced pancreatic cancer (SCALOP): A multicentre, randomised, phase 2 trial. Lancet Oncol. 2013, 14, 317-326. [CrossRef]

83. Hurt, C.N.; Falk, S.; Crosby, T.; McDonald, A.; Ray, R.; Joseph, G.; Staffurth, J.; Abrams, R.A.; Griffiths, G.; Maughan, T.; et al. Long-term results and recurrence patterns from SCALOP: A phase II randomised trial of gemcitabine- or capecitabine-based chemoradiation for locally advanced pancreatic cancer. Br. J. Cancer 2017, 116, 1264-1270. [CrossRef] [PubMed]

84. Krishnan, S.; Rana, V.; Janjan, N.A.; Varadhachary, G.R.; Abbruzzese, J.L.; Das, P.; Delclos, M.E.; Gould, M.S.; Evans, D.B.; Wolff, R.A.; et al. Induction chemotherapy selects patients with locally advanced, unresectable pancreatic cancer for optimal benefit from consolidative chemoradiation therapy. Cancer 2007, 110, 47-55. [CrossRef] [PubMed] 
85. Huguet, F.; André, T.; Hammel, P.; Artru, P.; Balosso, J.; Selle, F.; Deniaud-Alexandre, E.; Ruszniewski, P.; Touboul, E.; Labianca, R.; et al. Impact of Chemoradiotherapy After Disease Control with Chemotherapy in Locally Advanced Pancreatic Adenocarcinoma in GERCOR Phase II and III Studies. J. Clin. Oncol. 2007, 25, 326-331. [CrossRef] [PubMed]

86. Chang, J.S.; Chiu, Y.-F.; Yu, J.-C.; Chen, L.-T.; Ch'Ang, H.-J. The Role of Consolidation Chemoradiotherapy in Locally Advanced Pancreatic Cancer Receiving Chemotherapy: An Updated Systematic Review and Meta-Analysis. Cancer Res. Treat. 2018, 50, 562-574. [CrossRef] [PubMed]

87. Kasuga, A.; Hamamoto, Y.; Takeuchi, A.; Kawasaki, K.; Suzuki, T.; Hirata, K.; Sukawa, Y.; Takaishi, H.; Kanai, T. Positive relationship between subsequent chemotherapy and overall survival in pancreatic cancer: Meta-analysis of postprogression survival for first-line chemotherapy. Cancer Chemother. Pharmacol. 2017, 79, 595-602. [CrossRef] [PubMed]

88. Rahma, O.E.; Duffy, A.; Liewehr, D.J.; Steinberg, S.M.; Greten, T.F. Second-line treatment in advanced pancreatic cancer: A comprehensive analysis of published clinical trials. Ann. Oncol. 2013, 24, 1972-1979. [CrossRef]

89. Moss, A.C.; Morris, E.; Mac Mathuna, P. Palliative biliary stents for obstructing pancreatic carcinoma. Cochrane Database Syst. Rev. 2006, 169, 500.

90. Söderlund, C.; Linder, S. Covered metal versus plastic stents for malignant common bile duct stenosis: A prospective, randomized, controlled trial. Gastrointest. Endosc. 2006, 63, 986-995. [CrossRef]

91. Glazer, E.S.; Hornbrook, M.C.; Krouse, R.S. A meta-analysis of randomized trials: Immediate stent placement vs. surgical bypass in the palliative management of malignant biliary obstruction. J. Pain Symptom Manag. 2014, 47, 307-314. [CrossRef] [PubMed]

92. Jeurnink, S.M.; Van Eijck, C.H.; Steyerberg, E.W.; Kuipers, E.J.; Siersema, P.D. Stent versus gastrojejunostomy for the palliation of gastric outlet obstruction: A systematic review. BMC Gastroenterol. 2007, 7, 18. [CrossRef] [PubMed]

93. Gurusamy, K.S.; Kumar, S.; Davidson, B.R. Prophylactic gastrojejunostomy for unresectable periampullary carcinoma. Cochrane Database Syst. Rev. 2013, 6, CD008533. [CrossRef] [PubMed]

94. Zhong, W.; Yu, Z.; Zeng, J.X.; Lin, Y.; Yu, T.; Min, X.H.; Yuan, Y.H.; Chen, Q.K. Celiac plexus block for treatment of pain associated with pancreatic cancer: A meta-analysis. Pain Pract. 2014, 14, 43-51. [CrossRef] [PubMed]

95. Buwenge, M.; Macchia, G.; Arcelli, A.; Frakulli, R.; Fuccio, L.; Guerri, S.; Grassi, E.; Cammelli, S.; Cellini, F.; Morganti, A.G. Stereotactic radiotherapy of pancreatic cancer: A systematic review on pain relief. J. Pain Res. 2018, 11, 2169-2178. [CrossRef]

96. Gilliland, T.M.; Villafane-Ferriol, N.; Shah, K.P.; Shah, R.M.; Cao, H.S.T.; Massarweh, N.N.; Silberfein, E.J.; Choi, E.A.; Hsu, C.; McELHANY, A.L.; et al. Nutritional and Metabolic Derangements in Pancreatic Cancer and Pancreatic Resection. Nutrients 2017, 9, 243. [CrossRef]

97. Lee, A.Y.; Levine, M.N.; Baker, R.I.; Kakkar, A.K.; Prins, M.; Rickles, F.R.; Julian, J.A.; Haley, S.; Kovacs, M.J.; Bowden, C.; et al. Low-Molecular-Weight Heparin versus a Coumarin for the Prevention of Recurrent Venous Thromboembolism in Patients with Cancer. N. Engl. J. Med. 2003, 349, 146-153. [CrossRef]

98. Pelzer, U.; Opitz, B.; Deutschinoff, G.; Stauch, M.; Reitzig, P.C.; Hahnfeld, S.; Müller, L.; Grunewald, M.; Stieler, J.M.; Sinn, M.; et al. Efficacy of Prophylactic Low-Molecular Weight Heparin for Ambulatory Patients with Advanced Pancreatic Cancer: Outcomes from the CONKO-004 Trial. J. Clin. Oncol. 2015, 33, 2028-2034. [CrossRef]

99. Wormann, S.M.; Diakopoulos, K.N.; Lesina, M.; Algul, H. The immune network in pancreatic cancer development and progression. Oncogene 2014, 33, 2956-2967. [CrossRef]

100. Sideras, K.; Braat, H.; Kwekkeboom, J.; Van Eijck, C.; Peppelenbosch, M.; Sleijfer, S.; Bruno, M. Role of the immune system in pancreatic cancer progression and immune modulating treatment strategies. Cancer Treat. Rev. 2014, 40, 513-522. [CrossRef]

101. Garrido-Laguna, I.; Hidalgo, M. Pancreatic cancer: From state-of-the-art treatments to promising novel therapies. Nat. Rev. Clin. Oncol. 2015, 12, 319-334. [CrossRef] [PubMed]

102. Shoji, Y.; Miyamoto, M.; Ishikawa, K.; Yoshioka, T.; Mishra, R.; Ichinokawa, K.; Matsumura, Y.; Itoh, T.; Shinohara, T.; Hirano, S.; et al. The CD40-CD154 interaction would correlate with proliferation and immune escape in pancreatic ductal adenocarcinoma. J. Surg. Oncol. 2011, 103, 230-238. [CrossRef] [PubMed] 
103. Beatty, G.L.; Chiorean, E.G.; Fishman, M.P.; Saboury, B.; Teitelbaum, U.R.; Sun, W.; Huhn, R.D.; Song, W.; Li, D.; Sharp, L.L.; et al. CD40 Agonists Alter Tumor Stroma and Show Efficacy Against Pancreatic Carcinoma in Mice and Humans. Science 2011, 331, 1612-1616. [CrossRef] [PubMed]

104. Beatty, G.L.; Torigian, D.A.; Chiorean, E.G.; Saboury, B.; Brothers, A.; Alavi, A.; Troxel, A.B.; Sun, W.; Teitelbaum, U.R.; Vonderheide, R.H.; et al. A phase I study of an agonist CD40 monoclonal antibody (CP-870,893) in combination with gemcitabine in patients with advanced pancreatic ductal adenocarcinoma. Clin. Cancer Res. 2013, 19, 6286-6295. [CrossRef] [PubMed]

105. Nomi, T.; Sho, M.; Akahori, T.; Hamada, K.; Kubo, A.; Kanehiro, H.; Nakamura, S.; Enomoto, K.; Yagita, H.; Azuma, M.; et al. Clinical Significance and Therapeutic Potential of the Programmed Death-1 Ligand/Programmed Death-1 Pathway in Human Pancreatic Cancer. Clin. Cancer Res. 2007, 13, 2151-2157. [CrossRef] [PubMed]

106. Brahmer, J.R.; Tykodi, S.S.; Chow, L.Q.; Hwu, W.J.; Topalian, S.L.; Hwu, P.; Drake, C.G.; Camacho, L.H.; Kauh, J.; Odunsi, K.; et al. Safety and activity of anti-PD-L1 antibody in patients with advanced cancer. N. Engl. J. Med. 2012, 366, 2455-2465. [CrossRef]

107. Feig, C.; Jones, J.O.; Kraman, M.; Wells, R.J.B.; Deonarine, A.; Chan, D.S.; Connell, C.M.; Roberts, E.W.; Zhao, Q.; Caballero, O.L.; et al. Targeting CXCL12 from FAP-expressing carcinoma-associated fibroblasts synergizes with anti-PD-L1 immunotherapy in pancreatic cancer. Proc. Natl. Acad. Sci. USA 2013, 110, 20212-20217. [CrossRef]

108. Özdemir, B.C.; Pentcheva-Hoang, T.; Carstens, J.L.; Zheng, X.; Wu, C.C.; Simpson, T.R.; Laklai, H.; Sugimoto, H.; Kahlert, C.; Novitskiy, S.V.; et al. Depletion of carcinoma-associated fibroblasts and fibrosis induces immunosuppression and accelerates pancreas cancer with reduced survival. Cancer Cell 2014, 25, 719-734. [CrossRef]

109. Royal, R.E.; Levy, C.; Turner, K.; Mathur, A.; Hughes, M.; Kammula, U.S.; Sherry, R.M.; Topalian, S.L.; Yang, J.C.; Lowy, I.; et al. Phase 2 Trial of Single Agent Ipilimumab (Anti-CTLA-4) for Locally Advanced or Metastatic Pancreatic Adenocarcinoma. J. Immunother. 2010, 33, 828-833. [CrossRef]

110. Alvarez, R.; Musteanu, M.; Garcia-Garcia, E.; Lopez-Casas, P.P.; Megias, D.; Guerra, C.; Muñoz, M.; Quijano, Y.; Cubillo, A.; Rodriguez-Pascual, J.; et al. Stromal disrupting effects of nab-paclitaxel in pancreatic cancer. Br. J. Cancer 2013, 109, 926-933. [CrossRef]

111. Wedén, S.; Klemp, M.; Gladhaug, I.P.; Møller, M.; Eriksen, J.A.; Gaudernack, G.; Buanes, T. Long-term follow-up of patients with resected pancreatic cancer following vaccination against mutant K-ras. Int. J. Cancer 2011, 128, 1120-1128. [CrossRef] [PubMed]

112. Abou-Alfa, G.K.; Chapman, P.B.; Feilchenfeldt, J.; Brennan, M.F.; Capanu, M.; Gansukh, B.; Jacobs, G.; Levin, A.; Neville, D.; Kelsen, D.P.; et al. Targeting Mutated K-ras in Pancreatic Adenocarcinoma Using an Adjuvant Vaccine. Am. J. Clin. Oncol. 2011, 34, 321-325. [CrossRef] [PubMed]

113. Jaffee, E.M.; Hruban, R.H.; Biedrzycki, B.; Laheru, D.; Schepers, K.; Sauter, P.R.; Goemann, M.; Coleman, J.; Grochow, L.; Donehower, R.C.; et al. Novel Allogeneic Granulocyte-Macrophage Colony-Stimulating Factor-Secreting Tumor Vaccine for Pancreatic Cancer: A Phase I Trial of Safety and Immune Activation. J. Clin. Oncol. 2001, 19, 145-156. [CrossRef] [PubMed]

114. Lutz, E.; Yeo, C.J.; Lillemoe, K.D.; Biedrzycki, B.; Kobrin, B.; Herman, J.; Sugar, E.; Piantadosi, S.; Cameron, J.L.; Solt, S.; et al. A Lethally Irradiated Allogeneic Granulocyte-Macrophage Colony Stimulating Factor-Secreting Tumor Vaccine for Pancreatic Adenocarcinoma: A Phase II Trial of Safety, Efficacy, and Immune Activation. Ann. Surg. 2011, 253, 328-335. [PubMed]

115. Laheru, D.; Lutz, E.; Burke, J.; Biedrzycki, B.; Solt, S.; Onners, B.; Tartakovsky, I.; Nemunaitis, J.; Le, D.; Sugar, E.; et al. Allogeneic granulocyte macrophage colony-stimulating factor-secreting tumor immunotherapy alone or in sequence with cyclophosphamide for metastatic pancreatic cancer: A pilot study of safety, feasibility, and immune activation. Clin. Cancer Res. 2008, 14, 1455-1463. [CrossRef] [PubMed]

116. Le, D.T.; Brockstedt, D.G.; Nir-Paz, R.; Hampl, J.; Mathur, S.; Nemunaitis, J.; Sterman, D.H.; Hassan, R.; Lutz, E.; Moyer, B.; et al. A live-attenuated Listeria vaccine (ANZ-100) and a live-attenuated Listeria vaccine expressing mesothelin (CRS-207) for advanced cancers: Phase I studies of safety and immune induction. Clin. Cancer Res. 2012, 18, 858-868. [CrossRef] [PubMed] 
117. Le, D.T.; Wang-Gillam, A.; Picozzi, V.; Greten, T.F.; Crocenzi, T.; Springett, G.; Morse, M.; Zeh, H.; Cohen, D.; Fine, R.L.; et al. Safety and Survival with GVAX Pancreas Prime and Listeria Monocytogenes-Expressing Mesothelin (CRS-207) Boost Vaccines for Metastatic Pancreatic Cancer. J. Clin. Oncol. 2015, 33, 1325-1333. [CrossRef] [PubMed]

118. Le, D.T.; Lutz, E.; Uram, J.N.; Sugar, E.A.; Onners, B.; Solt, S.; Zheng, L.; Diaz, L.A.; Donehower, R.C.; Jaffee, E.M.; et al. Evaluation of Ipilimumab in combination with allogeneic pancreatic tumor cells transfected with a GM-CSF gene in previously treated pancreatic cancer. J. Immunother. 2013, 36, 382-389. [CrossRef] [PubMed]

119. Lutz, E.R.; Wu, A.A.; Bigelow, E.; Sharma, R.; Mo, G.; Soares, K.; Solt, S.; Dorman, A.; Wamwea, A.; Yager, A.; et al. Immunotherapy Converts Non-immunogenic Pancreatic Tumors into Immunogenic Foci of Immune Regulation. Cancer Immunol. Res. 2014, 2, 616-631. [CrossRef]

120. Middleton, G.; Silcocks, P.; Cox, T.; Valle, J.; Wadsley, J.; Propper, D.; Coxon, F.; Ross, P.; Madhusudan, S.; Roques, T.; et al. Gemcitabine and capecitabine with or without telomerase peptide vaccine GV1001 in patients with locally advanced or metastatic pancreatic cancer (TeloVac): An open-label, randomised, phase 3 trial. Lancet Oncol. 2014, 15, 829-840. [CrossRef]

121. Wood, L.D.; Hruban, R.H. Pathology and Molecular Genetics of Pancreatic Neoplasms. Cancer J. 2012, 18, 492-501. [CrossRef] [PubMed]

122. Eser, S.; Schnieke, A.; Schneider, G.; Saur, D. Oncogenic KRAS signalling in pancreatic cancer. Br. J. Cancer 2014, 111, 817-822. [CrossRef] [PubMed]

123. Ying, H.; Zhang, H.; Coloff, J.L.; Yan, H.; Wang, W.; Chen, S.; Viale, A.; Zheng, H.; Paik, J.-H.; Lim, C.; et al. Abstract A101: Oncogenic Kras maintains pancreatic tumors through regulation of anabolic glucose metabolism. Tumor Biol. 2012, 72, A101.

124. Collins, M.A.; Bednar, F.; Zhang, Y.; Brisset, J.-C.; Galbán, S.; Galbán, C.J.; Rakshit, S.; Flannagan, K.S.; Adsay, N.V.; Di Magliano, M.P. Oncogenic Kras is required for both the initiation and maintenance of pancreatic cancer in mice. J. Clin. Investig. 2012, 122, 639-653. [CrossRef] [PubMed]

125. Collins, M.A.; Brisset, J.-C.; Zhang, Y.; Bednar, F.; Pierre, J.; Heist, K.A.; Galbán, C.J.; Galbán, S.; Di Magliano, M.P. Metastatic Pancreatic Cancer Is Dependent on Oncogenic Kras in Mice. PLoS ONE 2012, 7, e49707. [CrossRef] [PubMed]

126. Kanda, M.; Matthaei, H.; Wu, J.; Hong, S.; Yu, J.; Borges, M.; Hruban, R.H.; Maitra, A.; Kinzler, K.; Vogelstein, B.; et al. Presence of Somatic Mutations in Most Early-Stage Pancreatic Intraepithelial Neoplasia. Gastroenterology 2012, 142, 730-733.e9. [CrossRef] [PubMed]

127. Morris, J.P.; Wang, S.C.; Hebrok, M. KRAS, Hedgehog, Wnt and the twisted developmental biology of pancreatic ductal adenocarcinoma. Nat. Rev. Cancer 2010, 10, 683-695. [CrossRef] [PubMed]

128. Oshima, M.; Okano, K.; Muraki, S.; Haba, R.; Maeba, T.; Suzuki, Y.; Yachida, S. Immunohistochemically Detected Expression of 3 Major Genes (CDKN2A/p16, TP53, and SMAD4/DPC4) Strongly Predicts Survival in Patients with Resectable Pancreatic Cancer. Ann. Surg. 2013, 258, 336-346. [CrossRef] [PubMed]

129. Caldas, C.; Hahn, S.A.; Da Costa, L.T.; Redston, M.S.; Schutte, M.; Seymour, A.B.; Weinstein, C.L.; Hruban, R.H.; Yeo, C.J.; Kern, S.E. Frequent somatic mutations and homozygous deletions of the p16 (MTS1) gene in pancreatic adenocarcinoma. Nat. Genet. 1994, 8, 27-32. [CrossRef] [PubMed]

130. Schutte, M.; Hruban, R.H.; Geradts, J.; Maynard, R.; Hilgers, W.; Rabindran, S.K.; Moskaluk, C.A.; Hahn, S.A.; Schwarte-Waldhoff, I.; Schmiegel, W.; et al. Abrogation of the Rb/p16 tumor-suppressive pathway in virtually all pancreatic carcinomas. Cancer Res. 1997, 57, 3126-3130.

131. Maitra, A.; Adsay, N.V.; Argani, P.; Iacobuzio-Donahue, C.; De Marzo, A.; Cameron, J.L.; Yeo, C.J.; Hruban, R.H. Multicomponent Analysis of the Pancreatic Adenocarcinoma Progression Model Using a Pancreatic Intraepithelial Neoplasia Tissue Microarray. Mod. Pathol. 2003, 16, 902-912. [CrossRef] [PubMed]

132. Hosoda, W.; Chianchiano, P.; Griffin, J.F.; Pittman, M.E.; Brosens, L.A.A.; Noë, M.; Yu, J.; Shindo, K.; Suenaga, M.; Rezaee, N.; et al. Genetic analyses of isolated high-grade pancreatic intraepithelial neoplasia (HG-PanIN) reveal paucity of alterations in TP53 and SMAD4. J. Pathol. 2017, 242, 16-23. [CrossRef] [PubMed]

133. Scarpa, A.; Capelli, P.; Mukai, K.; Zamboni, G.; Oda, T.; Iacono, C.; Hirohashi, S. Pancreatic adenocarcinomas frequently show p53 gene mutations. Am. J. Pathol. 1993, 142, 1534-1543. [PubMed]

134. Ahmed, S.; Bradshaw, A.D.; Gera, S.; Dewan, M.Z.; Xu, R. The TGF-beta/Smad4 Signaling Pathway in Pancreatic Carcinogenesis and Its Clinical Significance. J. Clin. Med. 2017, 6, 5. [CrossRef] [PubMed] 
135. Bardeesy, N.; Cheng, K.-H.; Berger, J.H.; Chu, G.C.; Pahler, J.; Olson, P.; Hezel, A.F.; Horner, J.; Lauwers, G.Y; Hanahan, D.; et al. Smad4 is dispensable for normal pancreas development yet critical in progression and tumor biology of pancreas cancer. Genome Res. 2006, 20, 3130-3146. [CrossRef] [PubMed]

136. Ijichi, H.; Chytil, A.; Gorska, A.E.; Aakre, M.E.; Fujitani, Y.; Fujitani, S.; Wright, C.V.; Moses, H.L. Aggressive pancreatic ductal adenocarcinoma in mice caused by pancreas-specific blockade of transforming growth factor-beta signaling in cooperation with active Kras expression. Genes Dev. 2006, 20, 3147-3160. [CrossRef]

137. Zhou, H.; Telonis, A.G.; Jing, Y.; Xia, N.L.; Biederman, L.; Jimbo, M.; Blanco, F.; Londin, E.; Brody, J.R.; Rigoutsos, I. GPRC5A is a potential oncogene in pancreatic ductal adenocarcinoma cells that is upregulated by gemcitabine with help from HuR. Cell Death Dis. 2016, 7, e2294. [CrossRef]

138. Liu, B.; Yang, H.; Pilarsky, C.; Weber, G.F. The Effect of GPRC5a on the Proliferation, Migration Ability, Chemotherapy Resistance, and Phosphorylation of GSK-3beta in Pancreatic Cancer. Int. J. Mol. Sci. 2018, 19, 1870. [CrossRef]

139. Seifert, L.; Werba, G.; Tiwari, S.; Giao, L.N.N.; Alqunaibit, D.; Alothman, S.; Daley, D.; Hundeyin, M.; Mani, V.R.; Barilla, R.; et al. Abstract B20: The necrosome promotes pancreatic Oncogenesis via CXCL1 and mincle-induced immune Suppression. Mol. Driv. Pancreat. Cancer Biol. Metastasis 2016, 76, B20.

140. Pilarski, R. The Role of BRCA Testing in Hereditary Pancreatic and Prostate Cancer Families. Am. Soc. Clin. Oncol. Educ. Book 2019, 39, 79-86. [CrossRef]

141. Jones, S.; Zhang, X.; Parsons, D.W.; Lin, J.C.-H.; Leary, R.J.; Angenendt, P.; Mankoo, P.; Carter, H.; Kamiyama, H.; Jimeno, A.; et al. Core Signaling Pathways in Human Pancreatic Cancers Revealed by Global Genomic Analyses. Science 2008, 321, 1801-1806. [CrossRef] [PubMed]

142. Biankin, A.V.; Waddell, N.; Kassahn, K.S.; Gingras, M.-C.; Muthuswamy, L.B.; Johns, A.L.; Miller, D.K.; Wilson, P.J.; Patch, A.-M.; Wu, J.; et al. Pancreatic cancer genomes reveal aberrations in axon guidance pathway genes. Nature 2012, 491, 399-405. [CrossRef] [PubMed]

143. Network CGAR. Integrated Genomic Characterization of Pancreatic Ductal Adenocarcinoma. Cancer Cell 2017, 32, 185-203. [CrossRef] [PubMed]

144. Collisson, E.A.; Sadanandam, A.; Olson, P.; Gibb, W.J.; Truitt, M.; Gu, S.; Cooc, J.; Weinkle, J.; Kim, G.E.; Jakkula, L.; et al. Subtypes of Pancreatic Ductal Adenocarcinoma and Their Differing Responses to Therapy. Nat. Med. 2011, 17, 500-503. [CrossRef] [PubMed]

145. Moffitt, R.A.; Marayati, R.; Flate, E.L.; Volmar, K.E.; Loeza, S.G.H.; Hoadley, K.A.; Rashid, N.U.; Williams, L.A.; Eaton, S.C.; Chung, A.H.; et al. Virtual microdissection identifies distinct tumor- and stroma-specific subtypes of pancreatic ductal adenocarcinoma. Nat. Genet. 2015, 47, 1168-1178. [CrossRef] [PubMed]

146. Waddell, N.; Pajic, M.; Patch, A.-M.; Chang, D.K.; Kassahn, K.S.; Bailey, P.; Johns, A.L.; Miller, D.K.; Nones, K.; Quek, K.; et al. Whole genomes redefine the mutational landscape of pancreatic cancer. Nature 2015, 518, 495-501. [CrossRef] [PubMed]

147. Bailey, P.; Initiative, A.P.C.G.; Chang, D.K.; Nones, K.; Johns, A.L.; Patch, A.-M.; Gingras, M.-C.; Miller, D.K.; Christ, A.N.; Bruxner, T.J.C.; et al. Genomic analyses identify molecular subtypes of pancreatic cancer. Nature 2016, 531, 47-52. [CrossRef]

148. Notta, F.; Chan-Seng-Yue, M.; Lemire, M.; Li, Y.; Wilson, G.W.; Connor, A.A.; Denroche, R.E.; Liang, S.B.; Brown, A.M.; Kim, J.C.; et al. A renewed model of pancreatic cancer evolution based on genomic rearrangement patterns. Nature 2016, 538, 378-382. [CrossRef]

149. Murphy, S.J.; Hart, S.N.; Lima, J.F.; Kipp, B.R.; Klebig, M.; Winters, J.L.; Szabo, C.; Zhang, L.; Eckloff, B.W.; Petersen, G.M.; et al. Genetic Alterations Associated with Progression from Pancreatic Intraepithelial Neoplasia to Invasive Pancreatic Tumor. Gastroenterology 2013, 145, 1098-1109.e1. [CrossRef]

150. Silverman, B.R.; Shi, J. Alterations of Epigenetic Regulators in Pancreatic Cancer and Their Clinical Implications. Int. J. Mol. Sci. 2016, 17, 2138. [CrossRef]

151. Nones, K.; Waddell, N.; Song, S.; Patch, A.-M.; Miller, D.; Johns, A.; Wu, J.; Kassahn, K.S.; Wood, D.; Bailey, P.; et al. Genome-wide DNA methylation patterns in pancreatic ductal adenocarcinoma reveal epigenetic deregulation of SLIT-ROBO, ITGA2 and MET signaling. Int. J. Cancer 2014, 135, 1110-1118. [CrossRef] [PubMed]

152. Gao, J.; Wang, L.; Xu, J.; Zheng, J.; Man, X.; Wu, H.; Jin, J.; Wang, K.; Xiao, H.; Li, S.; et al. Aberrant DNA methyltransferase expression in pancreatic ductal adenocarcinoma development and progression. J. Exp. Clin. Cancer Res. 2013, 32, 86. [CrossRef] [PubMed] 
153. Zhang, J.-J.; Zhu, Y.; Zhu, Y.; Wu, J.-L.; Liang, W.-B.; Zhu, R.; Xu, Z.-K.; Du, Q.; Miao, Y. Association of increased DNA methyltransferase expression with carcinogenesis and poor prognosis in pancreatic ductal adenocarcinoma. Clin. Transl. Oncol. 2012, 14, 116-124. [CrossRef] [PubMed]

154. Jiang, W.; Wang, J.; Zhang, Y. Histone H3K27me3 demethylases KDM6A and KDM6B modulate definitive endoderm differentiation from human ESCs by regulating WNT signaling pathway. Cell Res. 2013, 23, 122-130. [CrossRef] [PubMed]

155. Ougolkov, A.V.; Bilim, V.N.; Billadeau, D.D. Regulation of pancreatic tumor cell proliferation and chemoresistance by the histone methyltransferase enhancer of zeste homologue 2. Clin. Cancer Res. 2008, 14, 6790-6796. [CrossRef]

156. Wei, Y.; Xia, W.; Zhang, Z.; Liu, J.; Wang, H.; Adsay, N.V.; Albarracin, C.; Yu, D.; Abbruzzese, J.L.; Mills, G.B.; et al. Loss of Trimethylation at Lysine 27 of Histone H3 Is a Predictor of Poor Outcome in Breast, Ovarian, and Pancreatic Cancers. Mol. Carcinog. 2008, 47, 701-706. [CrossRef]

157. Schneider, G.; Krämer, O.H.; Schmid, R.M.; Saur, D. Acetylation as a Transcriptional Control Mechanism-HDACs and HATs in Pancreatic Ductal Adenocarcinoma. J. Gastrointest. Cancer 2011, 42, 85-92. [CrossRef]

158. Hessmann, E.; Johnsen, S.A.; Siveke, J.T.; Ellenrieder, V. Epigenetic treatment of pancreatic cancer: Is there a therapeutic perspective on the horizon? Gut 2017, 66, 168-179. [CrossRef]

159. Taketo, K.; Konno, M.; Asai, A.; Koseki, J.; Toratani, M.; Satoh, T.; Doki, Y.; Mori, M.; Ishii, H.; Ogawa, K. The epitranscriptome m6A writer METTL3 promotes chemo- and radioresistance in pancreatic cancer cells. Int. J. Oncol. 2018, 52, 621-629. [CrossRef]

160. He, Y.; Hu, H.; Wang, Y.; Yuan, H.; Lu, Z.; Wu, P.; Liu, D.; Tian, L.; Yin, J.; Jiang, K.; et al. ALKBH5 Inhibits Pancreatic Cancer Motility by Decreasing Long Non-Coding RNA KCNK15-AS1 Methylation. Cell. Physiol. Biochem. 2018, 48, 838-846. [CrossRef]

161. Martínez-Useros, J.; Georgiev-Hristov, T.; Fernandez-Aceñero, M.J.; Borrero-Palacios, A.; Indacochea, A.; Guerrero, S.; Li, W.; Cebrián, A.; Del Pulgar, T.G.; Puime-Otin, A.; et al. UNR/CDSE1 expression as prognosis biomarker in resectable pancreatic ductal adenocarcinoma patients: A proof-of-concept. PLoS ONE 2017, 12, e0182044. [CrossRef] [PubMed]

162. Li, W.; Martinez-Useros, J.; Garcia-Carbonero, N.; Fernandez-Aceñero, M.J.; Ortega-Medina, L.; Garcia-Botella, S.; Perez-Aguirre, E.; Diez-Valladares, L.; Garcia-Foncillas, J. The Prognosis Value of PIWIL1 and PIWIL2 Expression in Pancreatic Cancer. J. Clin. Med. 2019, 8, 1275. [CrossRef] [PubMed]

(C) 2019 by the authors. Licensee MDPI, Basel, Switzerland. This article is an open access article distributed under the terms and conditions of the Creative Commons Attribution (CC BY) license (http://creativecommons.org/licenses/by/4.0/). 